\title{
Evidence for Low Black Hole Spin and Physically Motivated Accretion Models From Millimeter-vlbi Observations of Sagittarius A*
}

\section{Citation}

Broderick, Avery E., Vincent L. Fish, Sheperd S. Doeleman, and Abraham Loeb. 2011. “EVIDENCE FOR LOW BLACK HOLE SPIN AND PHYSICALLY MOTIVATED ACCRETION MODELS FROM MILLIMETER-VLBI OBSERVATIONS OF SAGITTARIUS A*." The Astrophysical Journal 735 (2): 110. https://doi.org/10.1088/0004-637x/735/2/110.

\section{Permanent link}

http://nrs.harvard.edu/urn-3:HUL.InstRepos:41393163

\section{Terms of Use}

This article was downloaded from Harvard University's DASH repository, and is made available under the terms and conditions applicable to Other Posted Material, as set forth at http:// nrs.harvard.edu/urn-3:HUL.InstRepos:dash.current.terms-of-use\#LAA

\section{Share Your Story}

The Harvard community has made this article openly available.

Please share how this access benefits you. Submit a story.

\section{Accessibility}




\title{
EVIDENCE FOR LOW BLACK HOLE SPIN AND PHYSICALLY MOTIVATED ACCRETION MODELS FROM MILLIMETER-VLBI OBSERVATIONS OF SAGITTARIUS A*
}

\author{
Avery E. Broderick ${ }^{1}$, Vincent L. Fish ${ }^{2}$, Sheperd S. Doeleman ${ }^{2}$, And Abraham Loeb ${ }^{3}$ \\ ${ }^{1}$ Canadian Institute for Theoretical Astrophysics, 60 St. George Street, Toronto, ON M5S 3H8, Canada; aeb@ cita.utoronto.ca \\ ${ }^{2}$ Massachusetts Institute of Technology, Haystack Observatory, Route 40, Westford, MA 01886, USA \\ ${ }^{3}$ Institute for Theory and Computation, Harvard University, Center for Astrophysics, 60 Garden Street, Cambridge, MA 02138, USA \\ Received 2010 November 11; accepted 2011 April 20; published 2011 June 23
}

\begin{abstract}
Millimeter very long baseline interferometry (mm-VLBI) provides the novel capacity to probe the emission region of a handful of supermassive black holes on sub-horizon scales. For Sagittarius A* (Sgr A*), the supermassive black hole at the center of the Milky Way, this provides access to the region in the immediate vicinity of the horizon. Broderick et al. have already shown that by leveraging spectral and polarization information as well as accretion theory, it is possible to extract accretion-model parameters (including black hole spin) from mm-VLBI experiments containing only a handful of telescopes. Here we repeat this analysis with the most recent mmVLBI data, considering a class of aligned, radiatively inefficient accretion flow (RIAF) models. We find that the combined data set rules out symmetric models for Sgr A*'s flux distribution at the $3.9 \sigma$ level, strongly favoring length-to-width ratios of roughly 2.4:1. More importantly, we find that physically motivated accretion flow models provide a significantly better fit to the mm-VLBI observations than phenomenological models, at the $2.9 \sigma$ level. This implies that not only is mm-VLBI presently capable of distinguishing between potential physical models for Sgr A*'s emission, but further that it is sensitive to the strong gravitational lensing associated with the propagation of photons near the black hole. Based upon this analysis we find that the most probable magnitude, viewing angle, and position angle for the black hole spin are $a=0.0^{+0.64+0.86}, \theta=68_{-20^{\circ}-28^{\circ}}$, and $\xi=-52^{\circ}{ }_{-15^{\circ}-24^{\circ}}$ east of north, where the errors quoted are the $1 \sigma$ and $2 \sigma$ uncertainties.
\end{abstract}

Key words: accretion, accretion disks - black hole physics - Galaxy: center - submillimeter: general - techniques: interferometric

Online-only material: color figures

\section{INTRODUCTION}

Despite being invoked to power a variety of energetic astrophysical phenomena, the detailed structure and dynamics of black hole accretion flows remain a central problem in astrophysics. Moreover, using electromagnetic observations to probe the structure and dynamics of the black hole spacetimes requires a substantial understanding of the physical processes that determine the fate of the accreting matter. Only recently has it become possible to probe this physics via large-scale computational simulations. Nevertheless, ab initio calculations are beyond our present capability, requiring numerous simplifying, and in some cases unphysical, assumptions. This is evidenced by the number of models proffered to explain the various properties of accreting black hole candidates. In turn, this ambiguity complicates efforts to use electromagnetic observations to probe the structure and dynamics of the spacetime surrounding the black hole.

By virtue of its proximity, the supermassive black hole at the center of the Milky Way, associated with the bright radio point source Sagittarius A* (Sgr A*), provides an unparalleled opportunity to study black hole accretion in detail. For this reason, Sgr A* may serve as an exemplar of the larger class of supermassive black holes specifically, and of black holes in general. Presently, the best estimates of the mass and distance of Sgr A* come from the observations of orbiting stars. These have yielded $M=4.3 \pm 0.5 \times 10^{6} M_{\odot}$ and $D=8.3 \pm 0.4 \mathrm{kpc}$, respectively, where both include the systematic uncertainties (Ghez et al. 2008; Gillessen et al. 2009b, 2009a). The mass is necessarily confined to within the periapse of nearby stars, giving a maximum radius of roughly $10^{2} \mathrm{AU} \simeq 3 \times 10^{3} \mathrm{GM} / \mathrm{c}^{2}$, ruling out many extended objects. These represent the best mass measurement for any known black hole to date.

In addition to the dynamical observations, a wealth of spectral and polarization data exists for Sgr $\mathrm{A}^{*}$. From these it is apparent that $\mathrm{Sgr} \mathrm{A}^{*}$ is unlike many active galactic nuclei (AGNs), being vastly underluminous, emitting a bolometric luminosity of roughly $10^{36} \mathrm{erg}$, approximately $10^{-9}$ of Eddington. This is especially small in light of the considerable amount of gas within the black hole's sphere of influence, presumably available for accretion (Loeb \& Waxman 2007; Cuadra et al. 2008). As a result it is widely accepted that $\mathrm{Sgr} \mathrm{A}^{*}$ 's accretion flow is qualitatively different from those in its active analogs, though perhaps indicative of the roughly $90 \%$ of black holes that are presently not in an active phase.

Nevertheless, the existing spectral and polarization data have produced a canonical set of components all models for Sgr A* include: populations of thermal and nonthermal electrons, nearly equipartition magnetic fields. Less certain is the structure of the emission region. This is evidenced by the variety of models that have been proposed (e.g., Narayan et al. 1998; Blandford \& Begelman 1999; Falcke \& Markoff 2000; Yuan et al. 2002, 2003; Loeb \& Waxman 2007). Despite being able to reproduce the observed features of $\mathrm{Sgr} \mathrm{A}^{*}$, these differ dramatically in the morphology of the emitting region. As a consequence, many of the theoretical ambiguities can be immediately addressed by direct probes of the spatial distribution of the emitting plasma surrounding the central supermassive black hole.

The spectrum of Sgr A* peaks near millimeter wavelengths, implying a transition from optically thick to optically thin 
emission. The location of this emission is currently debated, however, the presence of short-timescale variability at millimeter, near-infrared, and X-ray wavelengths implies that optically thin emission is dominated by contributions arising in the immediate vicinity of the black hole. Furthermore, at millimeter wavelengths the blurring due to interstellar electron scattering is subdominant. Thus, at wavelengths of $1.3 \mathrm{~mm}$ and below it is possible to image the emitting region surrounding Sgr A*.

Even with the strong gravitational lensing in the vicinity of the horizon, imaging the immediate vicinity of the black hole requires extraordinary resolutions. The silhouette cast by the horizon on the surrounding emission is roughly $53 \pm 2 \mu \mathrm{as}^{4}$ At the present time, this resolution is accessible only via millimeter-wavelength very long baseline interferometry (mmVLBI). VLBI observations of Sgr A* at $1.4 \mathrm{~mm}$ using the Institut de Radioastronomie Millimétrique $30 \mathrm{~m}$ telescope at Pico Veleta and one of the $15 \mathrm{~m}$ dishes at Plateau de Bure produced the size estimate of $110 \pm 60 \mu \mathrm{as}$, with the large uncertainties due to limited calibration accuracy (Krichbaum et al. 1998).

The first successful mm-VLBI observation of Sgr A* with Earth-scale baselines was performed in 2007 April, during which visibilities were measured on the $4.6 \times 10^{3} \mathrm{~km}$ baseline between Mauna Kea, Hawaii and Mount Graham, Arizona (Doeleman et al. 2008). By fitting these with a Gaussian model, Doeleman et al. (2008) found a typical intrinsic source size of $37_{-3}^{+5} \mu$ as (after correcting for the subdominant broadening due to interstellar electron scattering $)^{5}$, smaller than the black hole silhouette.

Since that time a number of groups have analyzed the 2007 $\mathrm{mm}$-VLBI data using various physically motivated accretion models for the emission region (Broderick et al. 2009; Huang et al. 2009; Mościbrodzka et al. 2009; Dexter et al. 2010), inferring from these efforts the black hole spin vector. Despite finding generally similar results, these have been limited by the lack of multiple long-baseline observations and the limited north-south coverage obtained. Recently, a second and considerably larger set of mm-VLBI observations have been reported (Fish et al. 2011), providing the opportunity to revisit, and substantially improve, constraints upon the black hole spin and accretion physics.

Here we report upon the first effort to do this using a physically motivated accretion model, similar to that described in Broderick et al. (2009), that fits the known spectral and polarization properties of Sgr $\mathrm{A}^{*}$. In addition to improving the resulting parameter estimation, it is now possible to identify statistical signatures of both the asymmetry of the image and the importance of the underlying physics that governs the image morphology. Section 2 summarizes the full set of mm-VLBI observations we consider. Section 3 describes the models we consider and how the resulting visibility data are produced. How models are compared and the parameter estimates are produced is discussed in Section 4. The fitting process and results are presented in Section 5, and our best estimates for the black hole spin vector can be found in Section 6. Section 7 describes the implications for different potential future observations. Finally concluding remarks are collected in Section 8.

\footnotetext{
4 The mass and distance measurements are strongly correlated, with mass scaling roughly as $M \propto D^{1.8}$ (Ghez et al. 2008).

5 We quote the $1 \sigma$ errors implied by the $3 \sigma$ errors reported in Doeleman et al. (2008).
}

\section{SUMMARY OF MILLIMETER-VLBI OBSERVATIONS}

In the analysis presented here we make full use of the recent observations described in Fish et al. (2011) and Doeleman et al. (2008). In both cases, observations targeting Sgr A* were made at $1.3 \mathrm{~mm}$ using the Submillimeter Telescope (SMT) on Mt. Graham in Arizona, $10 \mathrm{~m}$ dishes in the Combined Array for Research in Millimeter-wave Astronomy (CARMA) at Cedar Flat, California, and the James Clerk Maxwell Telescope (JCMT) located on Mauna Kea, Hawaii.

\subsection{April}

Doeleman et al. (2008) report upon measurements obtained on the nights of the 2007 April 11 and 12, using the JCMT, SMT, and a single CARMA dish. 19 visibility amplitudes were obtained on the CARMA-SMT and JCMT-SMT baselines, with an upper limit on 2007 April 11, along the JCMT-CARMA baseline. The locations of these observations on the $u-v$ plane are indicated in the lower-left panel of Figure 1, labeled 2007. Signal-to-noise ratios typical of the short and long baselines are 8 and 4 , respectively.

During this time, observations the single-dish flux was estimated via the full CARMA array, operating as a stand-alone instrument, to be $2.4 \pm 0.25 \mathrm{Jy}$. This is similar to the visibility amplitudes obtained on the CARMA-SMT baselines and consistent with a single, compact Gaussian component (Doeleman et al. 2008). This flux is anomalously low in comparison to the typical $1.3 \mathrm{~mm}$ flux of $\sim 3 \mathrm{Jy}$, and was taken as evidence for Sgr A* appearing in a quiescent state. This interpretation is supported by the lack of a significant difference between analyses of each day separately (Broderick et al. 2009).

Full details of the observations, calibration, and data processing can be found in Doeleman et al. (2008).

\subsection{April}

Fish et al. (2011) report upon more recent observations performed on the nights of 2009 April 5-7, corresponding to the 95, 96, and 97 days of 2009. These made use of the JCMT, SMT, and two CARMA dishes, operated as independent VLBI stations. 54 visibility amplitudes were obtained on JCMT-SMT and CARMA-SMT baselines on all days, and to both of the JCMT-CARMA baselines on days 96 and 97. Positions of the observations on each day are indicated in the upper panels of Figure 1, labeled 2009.95, 2009.96, and 2009.97. Signal-tonoise ratios typical of the short and long baselines are 17 and 5, respectively. Thus, this second data set represents a significant improvement in both the number and precision of the data obtained.

In addition to the VLBI baselines, the presence of two independent CARMA dishes in the array allowed the measurement of very short baseline visibilities, probing angular scales $\sim 10^{\prime \prime}$. These found substantially more correlated flux density than the CARMA-SMT baselines did, inconsistent with a single compact Gaussian component. The interpretation of the difference in correlated flux density between the inter-CARMA baselines and the CARMA-SMT baselines is presently unclear, and it may be possible for multiple geometric models (e.g., annular rings, extended double source) to fit the data. Within the context of our analysis, we will assume that this difference is due to a separate large-scale component not present during the 2007 observations. This is indirectly supported by the fact that the source sizes inferred from the intermediate and long baseline 

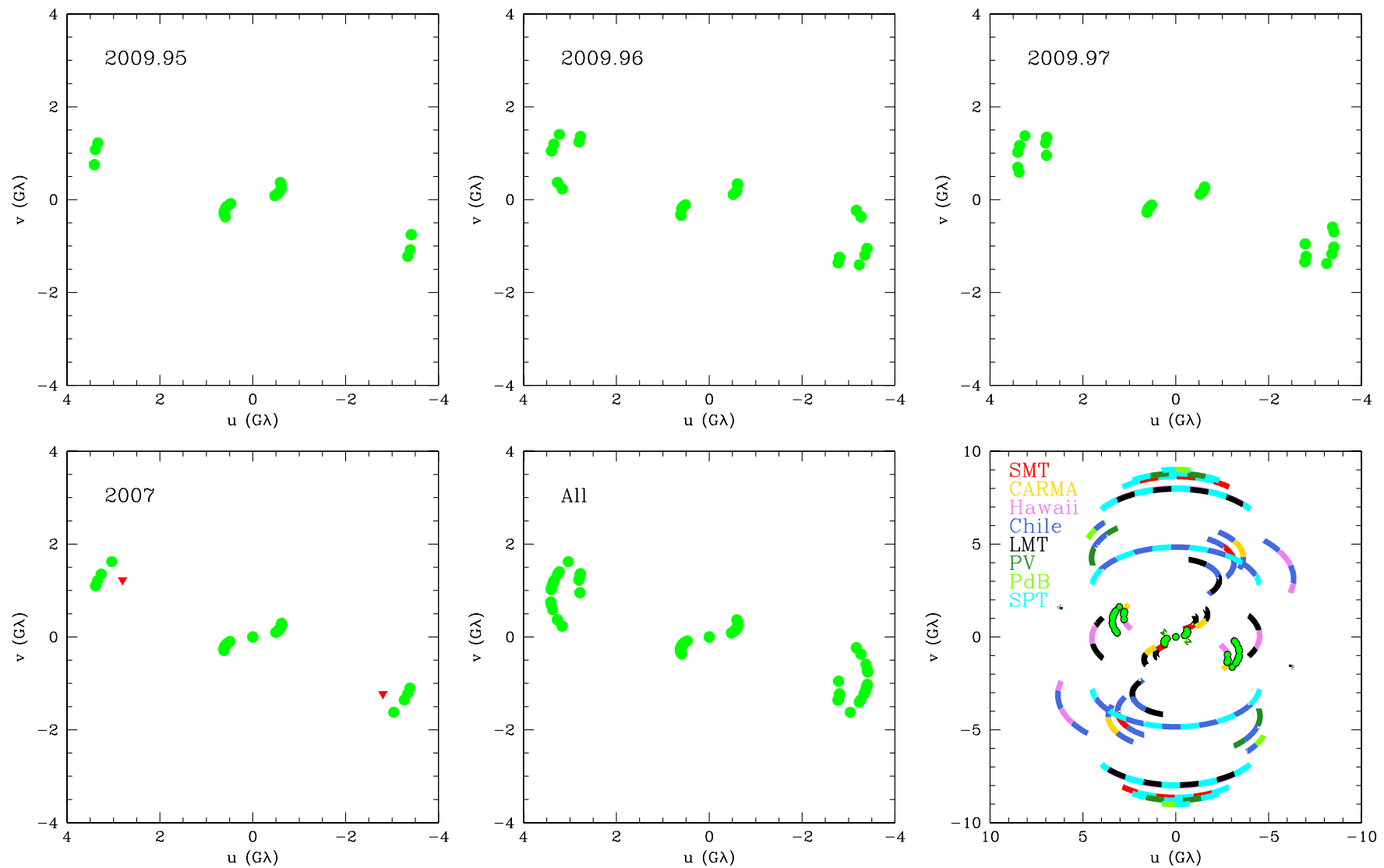

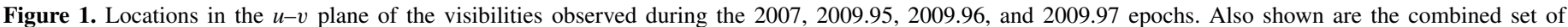

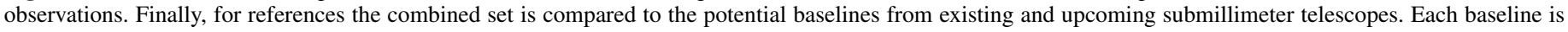
color-coded according to the associated two sites. In all plots, detections are denoted by green circles and upper limits by red triangles.

(A color version of this figure is available in the online journal.)

data are unchanged despite the variations in the visibility magnitudes (Fish et al. 2011). Therefore, we do not consider the inter-CARMA data further here, restricting ourselves to modeling the compact component observed with the longer baselines.

On days 95 and 96 the short-baseline flux densities are consistent with each other, with inferred single-dish fluxes of $2.15 \pm 0.06 \mathrm{Jy}$, which while somewhat lower than those obtained in 2007 justify treating these as a similar quiescent period. This is not the case for day 97, which exhibited a $30 \%-40 \%$ increase in the luminosity of the compact component. Note that during the $3 \mathrm{hr}$ observing periods on days 96 and 97 there is no evidence for rapid changes in the CARMA-SMT visibility amplitudes, implying that during each Sgr A* was stable; i.e., the process responsible for the brightening occurred between observing periods and is stable on timescales of hours. As a consequence, we will treat the visibilities obtained on each day as due to a stationary source, though with properties that vary from day to day.

Full details of the observations, calibration, and data processing can be found in Fish et al. (2011).

\subsection{Combined Data Set}

Combined, the 2007 and 2009 mm-VLBI measurements may be separated into four observational epochs: that containing the entire 2007 observations (2007), those on day 95 of 2009 (2009.95), those on day 96 of 2009 (2009.96), and those on day 97 of 2009 (2009.97). The combined coverage in the $u-v$ plane is shown in the lower-middle panel of Figure 1. The long baselines (JCMT-CARMA and JCMT-SMT) are oriented primarily in the east-west direction, extended roughly $3.6 \mathrm{G} \lambda$. Nevertheless, the combined data set also extends roughly $2 \mathrm{G} \lambda$ in the north-south direction, providing substantial angular coverage in the $u-v$ plane for the first time.

In Section 7 we will discuss the implications our analysis has for future observations. However, we note here that the baselines considered in the 2007 and 2009 mm-VLBI experiments are a small subset of the baselines that are possible with existing millimeter and submillimeter telescopes. Figure 1 shows the combined visibility data set in comparison to baselines associated with other potential mm-VLBI stations. These include stations in Chile (e.g., the Atacama Pathfinder EXperiment, Atacama Submillimeter Telescope Experiment, and Atacama Large Millimeter Array; APEX, ASTE, and ALMA, respectively), Mexico (Large Millimeter Telescope; LMT), Spain (Pico Veleta; PV), France (Plateau de Bure; PdB), and at the South Pole (South Pole Telescope; SPT). These both extend the region covered in the $u-v$ plane and provide additional complementary short and intermediate baselines, primarily along the north-south directions. To date, visibilities on only a handful of potential baselines have been measured.

\section{VISIBILITY MODELING}

Our primary goal is to use physically motivated models of Sgr A*'s accretion flow to infer the properties of the central supermassive black hole and its surrounding matter. To do this we compare both physical and phenomenological models of Sgr 
$\mathrm{A}^{*}$ to the mm-VLBI visibilities. This requires the computation of model visibilities. Given a trial image intensity distribution, $I(\alpha, \beta)$, where $\alpha$ and $\beta$ are angular coordinates, we may compute the visibilities in the standard fashion:

$$
V(u, v)=\iint \mathrm{d} \alpha \mathrm{d} \beta e^{-2 \pi i(\alpha u+\beta v) / \lambda} I(\alpha, \beta) .
$$

Here we describe three classes of model images: those associated with radiatively inefficient accretion flows (RIAFs) of the form discussed in Broderick \& Loeb (2006a), symmetric, and asymmetric Gaussians. We also summarize the effects of interstellar electron scattering.

\subsection{Radiatively Inefficient Accretion Flows}

We employ a suite of RIAF models, first described in Broderick \& Loeb (2006a) and based upon those of Yuan et al. (2003). Here these models, which henceforth we refer to as BL06, are summarized.

Sgr $A^{*}$ transitions from an inverted, presumably optically thick spectrum to an optically thin spectrum near millimeter wavelengths. This implies that near $1.3 \mathrm{~mm} \mathrm{Sgr} \mathrm{A}^{*}$ is only becoming optically thin, and thus absorption in the surrounding medium is likely to be important. This transition does not occur isotropically, happening at longer wavelengths for gas that is receding and at shorter wavelengths for gas that is approaching. Therefore, properly modeling the structure and relativistic radiative transfer is crucial to producing high fidelity images.

Although Sgr A* is vastly sub-Eddington, its bolometric luminosity, roughly $10^{36} \mathrm{erg} \mathrm{s}^{-1}$, is still large in absolute terms. Like many AGNs, in the radio Sgr A* exhibits the nearly-flat, power-law spectrum associated with nonthermal synchrotron sources, with the power emitted $\left(\nu L_{v}\right)$ peaking at millimeter wavelengths. As a consequence, it has been widely accepted that $\mathrm{Sgr} \mathrm{A}^{*}$ is accretion powered, implying a minimum accretion rate of $10^{-10} M_{\odot} \mathrm{yr}^{-1}$. It is presently unclear how this emission is produced, evidenced by the variety of models that have been proposed (e.g., Narayan et al. 1998; Blandford \& Begelman 1999; Falcke \& Markoff 2000; Yuan et al. 2002, 2003; Loeb \& Waxman 2007). Models in which the emission arises directly from the accreting gas have been subsumed into the general class of RIAFs, defined by the generally weak coupling between the electrons, which radiate rapidly, and the ions, which efficiently convert gravitational potential energy into heat (Narayan et al. 1998). This coupling may be sufficiently weak to allow accretion rates substantially in excess of that required to explain the observed luminosity with a canonical AGN radiative efficiency of $10 \%$. However, the detection of linear polarization in Sgr A* above 100GHz (Aitken et al. 2000; Bower et al. 2001, 2003; Marrone et al. 2006) and subsequent measurements of the Faraday rotation measure (Macquart et al. 2006; Marrone et al. 2007) have implied that the accretion rate near the black hole is much less than the Bondi rate, requiring the existence of large-scale outflows (Agol 2000; Quataert \& Gruzinov 2000).

Relating the outflow to the properties of the accretion flow requires an ab initio calculation that is presently not possible. Nevertheless, a number of authors have studied this relationship in the context of a variety of simplifying assumptions, with largescale general-relativistic magnetohydrodynamic and radiativehydrodynamic simulations playing a central role (De Villiers et al. 2005; McKinney 2006; Hawley \& Krolik 2006; Beckwith et al. 2008; McKinney \& Blandford 2009; Tchekhovskoy et al. 2010; Dexter et al. 2010; Penna et al. 2010; Kurosawa
\& Proga 2009). In these it has been found that the structure and dynamics of the outflow critically depends upon the initial conditions. The applicability of the MHD prescription to Sgr $\mathrm{A}^{*}$ is still unclear, where the accretion rate is sufficiently low that non-MHD effects may become important (Sharma et al. 2006, 2007). More importantly, most of these approaches do not model the electron heating (beyond ad hoc prescriptions) and none model the production of nonthermal electrons (see, e.g., Mościbrodzka et al. 2009; Dexter et al. 2010; Shcherbakov et al. 2010). Furthermore, simulations are computationally expensive to produce. For these reasons we adopt a simple, self-similar model for the accretion flow which includes substantial mass loss.

For concreteness, as in Broderick \& Loeb (2006a), we follow Yuan et al. (2003) and employ a model in which the accretion flow has a Keplerian velocity distribution, a population of thermal electrons with density and temperature

$$
n_{e, \mathrm{th}}=n_{e, \mathrm{th}}^{0}\left(\frac{r}{r_{\mathrm{S}}}\right)^{-1.1} e^{-z^{2} / 2 \rho^{2}}
$$

and

$$
T_{e}=n_{e}^{0}\left(\frac{r}{r_{\mathrm{S}}}\right)^{-0.84}
$$

respectively, and a toroidal magnetic field in approximate $(\beta=10)$ equipartition with the ions (which are responsible for the majority of the pressure), i.e.,

$$
\frac{B^{2}}{8 \pi}=\beta^{-1} n_{e, \text { th }} \frac{m_{p} c^{2} r_{\mathrm{S}}}{12 r}
$$

In all of these, $r_{\mathrm{S}}=2 G M / c^{2}$ is the Schwarzschild radius, $\rho$ is the cylindrical radius, and $z$ is the vertical coordinate. Inside of the innermost-stable circular orbit (ISCO) we assume the gas is plunging upon ballistic trajectories. In principle, the plunging gas can still radiate, though in practice it contributes little to the overall emission due to the large radial velocities it develops. In the case of the thermal quantities the radial structure was taken from Yuan et al. (2003), and the vertical structure was determined by assuming that the disk height is comparable to $\rho$. Note that all of the models we employ necessarily have the spin aligned with the orbital angular momentum of the accretion flow. For the regions that dominate the millimeter emission, this assumption is well justified due to disk precession and viscous torques, though it may be violated at large distances.

Thermal electrons alone are incapable of reproducing the nearly flat spectrum of Sgr $A^{*}$ below $43 \mathrm{GHz}$. Thus, it is necessary to also include a nonthermal component. As with the thermal components, we adopt a self-similar model for a population of nonthermal electrons,

$$
n_{e, \text { nth }}=n_{e, \text { nth }}^{0}\left(\frac{r}{r_{\mathrm{S}}}\right)^{-2.02} e^{-z^{2} / 2 \rho^{2}}
$$

with a power-law distribution corresponding to a spectral index of 1.25 and cutoff below Lorentz factors of $10^{2}$ (consistent with Yuan et al. 2003). The radial power-law index was chosen to reproduce the low frequency spectrum of $\mathrm{Sgr} \mathrm{A}^{*}$, and is insensitive to the black hole properties due to the distant location of the long-wavelength emission.

The primary emission mechanism at the wavelengths of interest is synchrotron, arising from both the thermal and nonthermal 


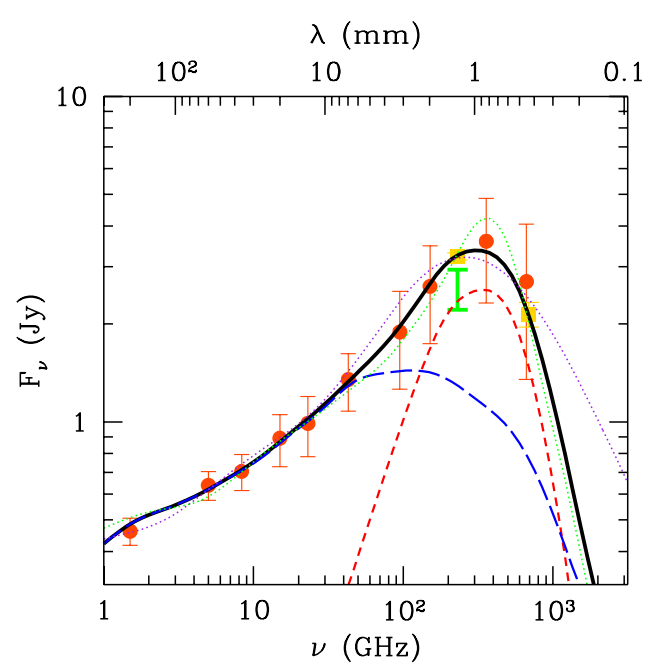

Figure 2. Comparison of the spectrum of the most probable accretion model and the observed SED of Sgr A*. Orange circles are from Yuan et al. (2004 and references therein), for which the error bars are indicative of the variability. Yellow squares are coincident flux measurements from Marrone (2006), for which the error bars are indicative of the intrinsic measurement error. The green bar shows the flux range of the compact component inferred from fitting accretion models to the mm-VLBI data (Section 5). In addition to the most probable model, spectra are shown for emission from a rapidly rotating black hole $(a=0.998)$ as seen nearly face-on $\left(\theta=1^{\circ}\right.$, green dotted) and edge-on $\left(\theta=90^{\circ}\right.$, purple dotted), indicating the range of variation within the image library. Finally, for reference the spectra associated when only the thermal (red dash) and nonthermal (blue long-dash) are present are shown. Note in particular that at $1.3 \mathrm{~mm}$ the nonthermal contribution is not negligible.

(A color version of this figure is available in the online journal.)

electrons. We model the emission from the thermal electrons using the emissivity described in Yuan et al. (2003), appropriately altered to account for relativistic effects (see, e.g., Broderick \& Blandford 2004). Since we perform polarized radiative transfer via the entire complement of Stokes parameters, we employ the polarization fraction for thermal synchrotron as derived in Petrosian \& McTiernan (1983). In doing so, we have implicitly assumed that the emission due to thermal electrons is isotropic, which while generally not the case is unlikely to change our results significantly. For the nonthermal electrons, we follow Jones \& O'Dell (1977) for a power-law electron distribution, with an additional spectral break associated with the minimum electron Lorentz factor. For both emission components the absorption coefficients are determined directly via Kirchhoff's law. Images are then produced using the fully relativistic ray-tracing and radiative transfer schemes described in Broderick \& Loeb (2006a, 2006b) and Broderick (2006). An example image and associated visibilities are shown in the bottom line of Figure 3.

Because Yuan et al. (2003) neglected relativistic effects and assumed spherical symmetry, it is not directly applicable here. For these reasons, as in Broderick \& Loeb (2006a), the coefficients $\left(n_{e, \text { th }}^{0}, T_{e}^{0}, n_{e, \text { nth }}^{0}\right)$ were adjusted to fit the radio spectral energy distribution (SED) of Sgr A*, shown in Figure 2. The $1.3 \mathrm{~mm}$ and $0.43 \mathrm{~mm}$ points (orange squares) were measured coincidentally, and the errors represent the intrinsic measurement error (Marrone 2006). All other points (red circles) are taken from Yuan et al. (2004 and references therein) and were obtained by averaging over multiple epochs. As a result, the error bars represent the range of variability and are correspondingly larger.

As in Broderick et al. (2009) we systematically fit Sgr A*'s SED at a large number of positions in the spin-inclination ( $a-\theta$, where $\theta$ is the viewing angle relative to the disk axis) parameter space, specifically at values of $a \in\{0,0.1,0.2, \ldots, 0.9,0.99,0.998\}$ for all $\theta \in$ $\left\{1^{\circ}, 10^{\circ}, 20^{\circ}, \ldots, 80^{\circ}, 90^{\circ}\right\}$, producing a tabulated set of the coefficients $\left(n_{e, \mathrm{th}}^{0}, T_{e}^{0}, n_{e, \mathrm{nth}}^{0}\right)$ at 120 points in the $a-\theta$ parameter space. In all cases it was possible to fit the SED with extraordinary precision, with reduced $-\chi^{2}<0.3$ in all cases and typically reduced- $\chi^{2} \simeq 0.17$. This is likely a consequence of employing the variability-determined error bars on the non-coincident flux measurements. Over the $a-\theta$ plane the $\chi^{2}$ was remarkably uniform, implying that on the basis of the spectra alone it is difficult to constrain the parameters of our simple model. Nevertheless, the dynamical properties of the disk manifest themselves in the breadth of the submillimeter bump. Models with low spin and/or low $\theta$ have little Doppler shifting, and correspondingly narrow bumps. In contrast, models with large spins $(a>0.9)$ viewed edge-on $\left(\theta>80^{\circ}\right)$ have broad bumps, and are responsible for the relatively larger, though still small, $\chi^{2}$. From the tabulated values, the coefficients are then obtained at arbitrary $a$ and $\theta$ using high-order polynomial interpolation.

An example spectrum, resulting from the above procedure, is shown in Figure 2, corresponding to $a=0$ and $\theta=68^{\circ}$. In addition, the spectra obtained when only the thermal and nonthermal components are independently considered are shown. Note that generally the spectrum cannot be decomposed in this fashion due to the nonlinearity of the radiative transfer equation. Nevertheless, the necessity of the nonthermal electrons is clearly illustrated at long wavelengths, where the thermal contribution is negligible. The transition from nonthermally dominated to thermally dominated occurs near $2 \mathrm{~mm}$, though the precise location depends upon $a$ and $\theta$. However, note that at no point can the nonthermal component be neglected. Specifically, at $1.3 \mathrm{~mm}$ the nonthermal component is still responsible for roughly $30 \%$ of the emission. As a result, efforts to model the millimeter image of Sgr A* without accounting for the nonthermal component can produce order unity systematic errors in parameter estimation.

During the mm-VLBI observations Sgr A*'s flux varied by roughly $30 \%$. We model this as a variable accretion rate, moving the electron density normalization up and down. In practice, we reduced the electron density normalization by an amount sufficient to produce a total flux of $2.5 \mathrm{Jy}$ and then multiplied the resulting images by a correction faction during the mm-VLBI data analysis. Because the source is not uniformly optically thin, this is not strictly correct, though this makes a small change to the images themselves. For the purpose of the mm-VLBI data analysis (described below) we produced 9090 images, with flux normalized as described above, at $a \in\{0,0.01,0.02, \ldots, 0.98,0.99,0.998\}$ for each $\theta \in\left\{1^{\circ}, 2^{\circ}, \ldots, 89^{\circ}, 90^{\circ}\right\}$. We then produce models with arbitrary position angles, $\xi$, by rotating the image on the sky. For this purpose we define $\xi$ such that at $0^{\circ}$ the projected spin vector points north, and as $\xi$ increases points progressively more eastward. ${ }^{6}$

The Faraday rotation measures observed in Sgr A* are produced at much larger radii than those of interest in direct imaging experiments. Nevertheless, it is worth noting that the models employed here are broadly consistent with the polarization observations, though breaks in the radial power laws which define the properties of the thermal electron component may be required at large spins.

\footnotetext{
6 Note that this is opposite of the definition employed in Broderick et al. (2009).
} 
For the purposes of fitting the mm-VLBI visibilities, during each observation epoch this model has four parameters: spin $(a)$, viewing angle $(\theta)$, position angle $(\xi)$, and flux normalization $\left(V_{00}\right)$. When we analyze multiple epochs together the parameters defining the orientation of the system $(a, \theta, \xi)$ will be held fixed, while those corresponding to the time-variable accretion rate $\left(V_{00}\right)$ will be allowed to vary, though a full discussion will have to await Section 5.

\subsection{Gaussian Flux Distributions}

For comparison we consider two Gaussian flux distributions. These differ from the accretion flow model described above in that they are purely phenomenological, without any clear physical motivation and thus not constrained at all by the spectral and polarization properties of Sgr A*. As a result, we might expect these to be intrinsically less likely than physically motivated models that are already chosen to be consistent with these properties. Nevertheless, we will consider them on equal footing with the BL06 model discussed above. For reasons that will become clear, we consider both symmetric and asymmetric Gaussian intrinsic flux distributions.

We may write the asymmetric Gaussian flux distribution as

$$
I=V_{00} \exp \left(-\frac{\alpha_{M}^{2}}{2 \sigma_{M}^{2}}-\frac{\alpha_{m}^{2}}{2 \sigma_{m}^{2}}\right)
$$

where $\alpha_{M, m}$ and $\sigma_{M, m}$ are the angular coordinates and widths in the major/minor axis directions. This is fully defined once $\sigma_{M, m}$ and the position angle of the major axis is given. However, we choose to parameterize the asymmetric Gaussian in terms of a single width, $\sigma$, an anisotropy parameter, $A$, and the position angle:

$$
I=V_{00} \exp \left(-\frac{\alpha^{2}}{2 \sigma^{2}}-A \frac{\alpha^{2}}{2 \sigma^{2}} \cos 2 \varpi\right),
$$

where $\alpha=\sqrt{\alpha_{M}^{2}+\alpha_{m}^{2}}$ and $\varpi$ is the angular coordinate measured from the position angle. The $\sigma$ and $A$ are related to the $\sigma_{M, m}$ by

$$
\frac{1}{\sigma^{2}}=\frac{1}{2 \sigma_{m}^{2}}+\frac{1}{2 \sigma_{M}^{2}} \quad \text { and } \quad \frac{A}{\sigma^{2}}=\frac{1}{2 \sigma_{m}^{2}}-\frac{1}{2 \sigma_{M}^{2}} .
$$

Example Gaussian images, with associated visibilities, are shown in the top two lines of Figure 3.

Clearly, for isotropic configurations (i.e., $\sigma_{M}=\sigma_{m}$ ), $A=0$ and $\sigma=\sigma_{M, m}$. More generally, $\sigma_{m} / \sigma_{M}=\sqrt{(1-A) /(1+A)}$. Thus, this parameterization has the virtue of separately emphasizing size (via $\sigma$ ) and asymmetry (via $A$ ) in the image. Note that this model has precisely the number of free parameters as the accretion flow model described above: those describing the image morphology, $(\sigma, A, \xi)$ and the flux normalization, $V_{00}$, for each epoch.

While the symmetric Gaussian models are obviously a subset of the asymmetric Gaussian models (corresponding to when $A$ vanishes), we must be careful to distinguish the number of free parameters. In this case, $A$ and $\xi$ are superfluous, and for each epoch we have only one parameter.

\subsection{Interstellar Electron Scattering}

The effects of interstellar electron scattering in the direction of $\operatorname{Sgr} \mathrm{A}^{*}$ have been carefully characterized empirically by a number of authors. This has been found to be consistent with convolving the source with an asymmetric Gaussian, with major axis nearly aligned with east-west, and a $\lambda^{2}$ wavelength dependence. We employ the model from Bower et al. (2006), which has major axis oriented $78^{\circ}$ east of north, with associated full width at half-maximum for the major and minor axes given by

$$
\begin{aligned}
\mathrm{FWHM}_{M}^{\mathrm{ES}} & =1.309\left(\frac{\lambda}{1 \mathrm{~cm}}\right)^{2} \mathrm{mas}, \\
\mathrm{FWHM}_{m}^{\mathrm{ES}} & =0.64\left(\frac{\lambda}{1 \mathrm{~cm}}\right)^{2} \text { mas }
\end{aligned}
$$

respectively. In practice, the interstellar electron scattering convolution was effected in the $u-v$ plane, where the convolution reduces to a multiplication.

\section{BAYESIAN DATA ANALYSIS}

In fitting the observed visibilities we have two primary goals: choosing among various possible model flux distributions and estimating the parameters of these models. Both of these may be naturally accomplished within the context of Bayesian analysis. Here we briefly summarize how we do this.

We define the likelihood, $p(\mathbf{V} \mid \mathbf{q})$, for observing the visibilities $\mathbf{V}$ given the model parameters $\mathbf{q}$, as described in Broderick et al. (2009). From this we obtain the log-likelihood, which we refer to as $\chi^{2}$ :

$$
\chi^{2} \equiv-2 \log p(\mathbf{V} \mid \mathbf{q})+C
$$

where the normalization constant depends only upon the particulars of the data and, since we will only be interested in comparing identical data sets, will henceforth be ignored. When only detections are considered this reduces trivially to the standard definition of $\chi^{2}$. Here it differs only due to the presence of an upper limit upon the visibility along the CARMA-JCMT baseline during the 2007 epoch. Ignoring this point produces negligible changes to the resulting log-likelihood and associated probabilities, and thus we may treat $\chi^{2}$ in the normal fashion for our purposes here.

\subsection{Model Comparison}

To compare the significance of different models we make use of the Bayesian Information Criterion (BIC) and the Akaike Information Criterion (AIC). Both of these are discussed in detail within the context of astrophysical observations by Liddle (2007) and Takeuchi (2000), and references therein. Thus, here we only define and summarize the properties of these statistics.

In terms of the smallest effective $\chi^{2}$ for a given model, the $\mathrm{BIC}$ is defined by

$$
\mathrm{BIC} \equiv \chi_{\min }^{2}+k \ln N,
$$

where $k$ is the number of model parameters and $N$ is the number of data points. Note that this is simply the $\chi^{2}$ statistic penalized for models with large numbers of parameters. Assuming that the data points are independent (likely true) and normally distributed (nearly true), this is related to the posterior probabilities of two different models, $M_{1}$ and $M_{2}$, by

$$
\frac{p\left(M_{1} \mid \mathbf{V}\right)}{p\left(M_{2} \mid \mathbf{V}\right)}=\frac{p\left(M_{1}\right)}{p\left(M_{2}\right)} e^{-\left(\mathrm{BIC}_{1}-\mathrm{BIC}_{2}\right) / 2}
$$



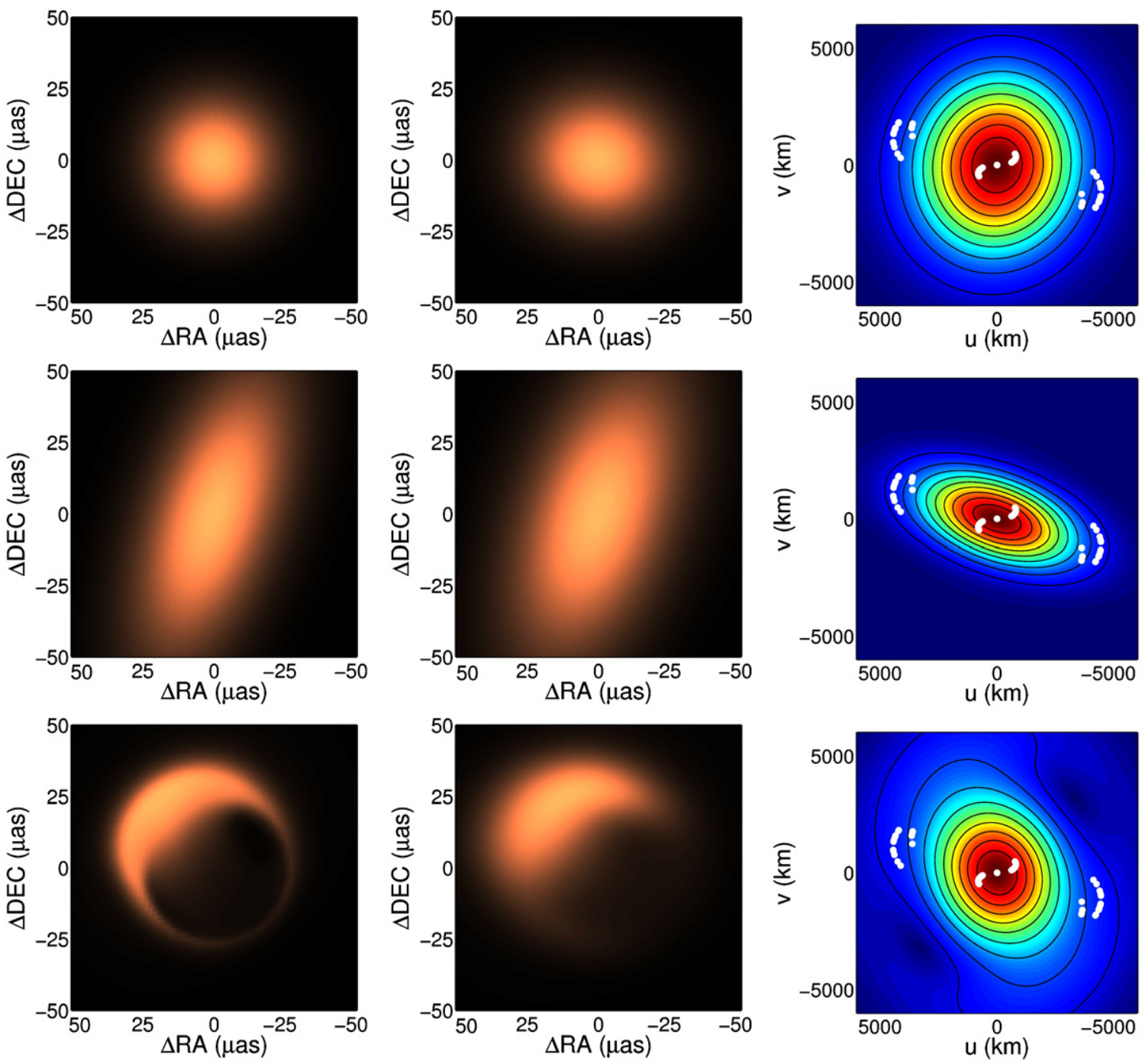

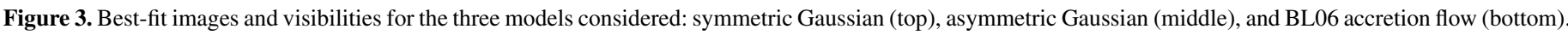

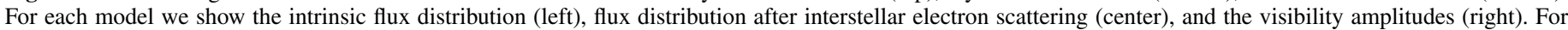
reference the locations of the observed visibilities (over all epochs) are shown by the white points. In all plots the intensity scales linearly with $I$ and $V$.

(A color version of this figure is available in the online journal.)

where $p\left(M_{1,2}\right)$ is the prior on model $M_{1,2}$. Thus, up to the unknown priors, the BIC is a measure of the posterior probability for a given model. If we further assume that $p\left(M_{1}\right)=p\left(M_{2}\right)$, the difference in BICs gives the relative posterior probabilities directly. Therefore, the model with the lowest BIC is preferred.

Similarly, the AIC is defined by

$$
\mathrm{AIC} \equiv \chi_{\min }^{2}+2 k+\frac{2 k(k+1)}{N-k-1}
$$

where we have included a correction appropriate for when $N$ is small (Burnham \& Anderson 2002, 2004). This is very similar to the BIC: the $\chi^{2}$ statistic penalized by a factor depending upon the number of model parameters, though with a somewhat different penalty. Unlike the BIC, the AIC is not directly related to the posterior probability of a given model. Rather it is an approximate measure of the difference between the true data distribution and the modeled data distribution. Nevertheless, it is possible to interpret the AIC in terms of a model likelihood in a way identical to the BIC.
For either criterion, lower values are preferred, with the relative significance given by

$$
w_{i j}=e^{-\left(\mathrm{IC}_{i}-\mathrm{IC}_{j}\right) / 2},
$$

where IC may be replaced by BIC or AIC. The $\triangle \mathrm{IC}$ are conventionally judged on the Jeffrey's scale, which sets $\Delta \mathrm{IC}>5$ as "strong" and $\Delta \mathrm{IC}>10$ as "decisive" evidence against the model with the higher IC. However, here we describe these in terms of the typical $\sigma$ as well, with model $i$ being excluded at the $n \sigma$ level if there exists a model $j$ for which $w_{i} j$ is less than the associated cumulative normal probability (e.g., $1 \sigma$ implies that $w_{i j}<0.32,2 \sigma$ implies that $w_{i j}<0.05,3 \sigma$ implies that $w_{i j}<0.003$, etc. $)$.

Note that the $w_{i} j$ are only defined for model pairs and represent the ratio of the equally weighted posterior probabilities. In principle, we could construct the probability that the $i$ th model is the true model: $W_{i} w_{i j} / \sum_{k} W_{k} w_{k j}$, where the $W_{i}$ are the priors on the $i$ th model, the sum is taken over all possible models, and $j$ is arbitrary. However, since the extent of the model space and associated priors are unknown, this is not possible in practice, and 
instead we will only quote the individual relative significances, $w_{i} j$.

While we will utilize the Bayesian scheme to perform model selection and physical parameter estimation, the $\chi^{2}$ statistic immediately allows us to address two important questions: quality of fit and an estimate of the uncertainties in the relative significance of models. While the Bayesian analysis allows us to rank models and parameter sets, it cannot independently assess the reasonability of a given fit. Thus, it is possible to end up ranking models that fail to reproduce the visibilities to varying degrees. We guard against this by checking that for every model we consider that some set of parameters exist for which the reduced- $\chi^{2}$ is of order unity, and thus good fits exist. Similarly, estimates of the visibility uncertainties may be obtained by assuming the true model and asserting that the reduced $-\chi^{2}=1$. This results in slight corrections to the BIC and $\mathrm{AIC}$, and therefore provides an estimate of the uncertainty in the relative significance of models.

\subsection{Parameter Estimation}

For the accretion flow model we have the additional problem of identifying the most likely model parameters. The procedure we use to estimate the posterior probabilities is identical to that described in Broderick et al. (2009). In particular, we assume flat priors on all of the visibility normalizations, $\mathbf{V}_{\mathbf{0 0}}$, the spin magnitude, $a$, and an isotropic prior on the spin direction $(\theta, \xi)$. Since we are primarily interested in the estimates for the black hole spin, we present the posterior probability of a marginalized over the $\mathbf{V}_{\mathbf{0 0}}, p(\mathbf{a})$. We also construct marginalized posterior probability distributions of $a, \theta$, and $\xi$ in the normal way (for specific definitions of $p(a), p(\theta)$, and $p(\xi)$; see Broderick et al. 2009).

For the phenomenological Gaussian models, for which the parameters do not have natural priors, we select the parameters and determine their errors via $\chi^{2}$ minimization and variations alone (in effect, choosing the priors to be flat).

\section{MODEL FITTING}

A number of important implications follow from computations of the relevant $\chi^{2}$ for the three image models described in Section 3. These include whether or not we are justified in comparing mm-VLBI observations obtained at different times, the symmetry of the image, and the importance of physics for reproducing the measured visibilities.

\subsection{Consistency of the 2007 and 2009 Epochs}

The dynamical timescale of the Sgr $\mathrm{A}^{*}$ is comparable to the orbital period at the ISCO, as measured at Earth, and ranges from roughly 4 minutes to 30 minutes, depending upon spin. As a result it is not at all clear that we may ignore the possibility of variability when attempting to model mm-VLBI observations spanning many nights, let alone years. Broderick et al. (2009) took special pains to ensure that the visibilities measured on the two consecutive days were consistent with a single, static underlying flux distribution. This was done by comparing fits to the individual days. Here we repeat this analysis for the 2009 observations as well.

In 2007 , at $2.4 \mathrm{Jy}$, the luminosity of Sgr A* was anomalously low and stable over the two observation days. This is not the case during the 2009 observation, during which Sgr A* exhibited a dramatic brightening on the third day. During the preceding two days the luminosity of Sgr A*'s compact component,

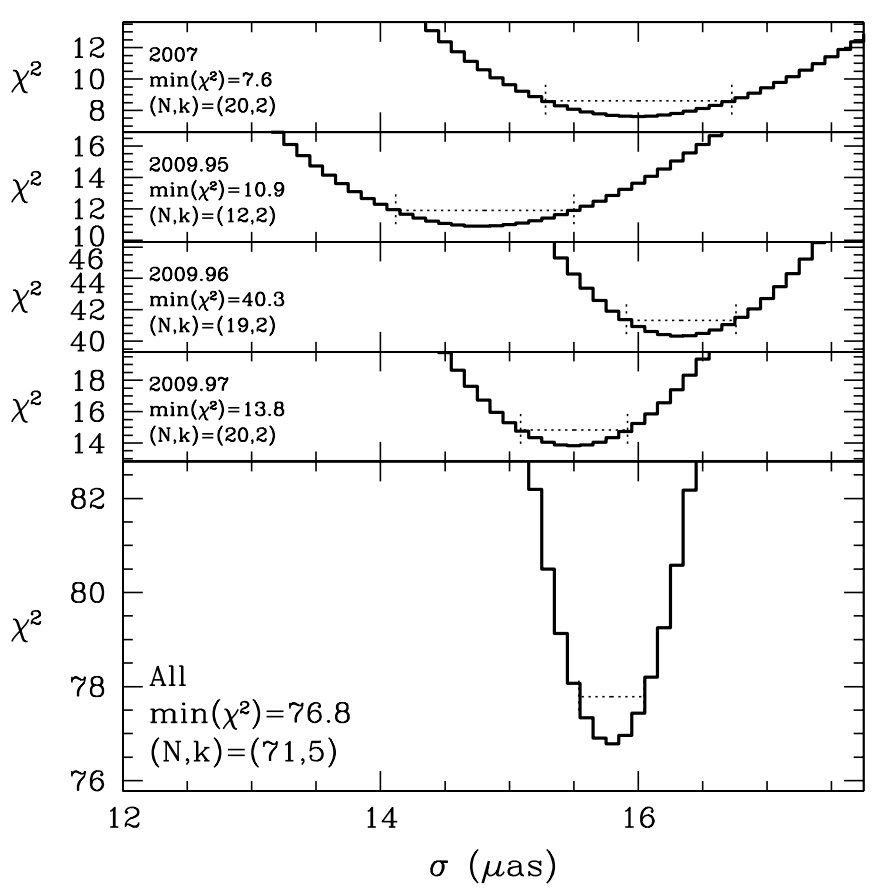

Figure 4. Symmetric Gaussian $\chi^{2}$ 's as a function of $\sigma$, at the most likely $\mathbf{V}_{\mathbf{0 0}}$, for the 2007, 2009.95, 2009.96, and 2009.97 epochs, as well as when all epochs are combined. The minimum $\chi^{2}$, number of visibility observations $(N)$, and fit parameters $(k)$ are listed in each plot. In all cases the range shown corresponds to $\min \left(\chi^{2}\right)-1$ to $\min \left(\chi^{2}\right)+6$. For references the $1 \sigma$ error estimate is shown by the dotted error bars for each data set.

corresponding to scales smaller than $10^{2} r_{\mathrm{S}}$, was significantly smaller than that associated with the 2007 observations. Thus, it is clear from the outset that we are not justified in comparing a single, static model to the observations. Instead, we begin with the ansatz that the morphology of Sgr A*'s image is fixed, with the flux variability being driven by changes in the accretion rate on day-to-day timescales. While this period is considerably larger than the 30 minute timescale over which the properties of the accretion flow may change, it is justified in part by the stability of Sgr $\mathrm{A}^{*}$ 's luminosity on these scales as well as the intrinsically short duration $(\sim 2 \mathrm{hr})$ of the observations each night. Therefore, we separate the data into four epochs: 2007, 2009.95, 2009.96, and 2009.97, corresponding to the data obtained in 2007 and on days 95, 96, and 97 of 2009, respectively. For all epochs we keep the parameters that define the image morphology fixed, e.g., $(a, \theta, \xi),(\sigma, A, \xi)$, or $\sigma$, but allow the overall flux normalization to vary from epoch to epoch. Upon fitting each epoch separately, and all epochs together, we may ask if the resulting parameter likelihoods are consistent with each other, i.e., check if our ansatz is self consistent. We will remark upon this further in the sections describing the fits for the individual models; however, here it is sufficient to note that in all cases we find that the epochs are indeed consistent with a single underlying image morphology.

\subsection{Symmetric Gaussian}

We begin with the symmetric Gaussian model, which is primarily sensitive to the characteristic size of the image. In this case for each epoch there are two parameters: $\sigma$ and $V_{00}$. The minimum $\chi^{2}$ is shown as a function of $\sigma$ for each epoch in Figure 4. With the exception of epoch 2009.96, the reduced $-\chi^{2}$ is comparable to unity. Over all epochs the $\sigma$ with the highest likelihood varies over $1.5 \mu \mathrm{as}$, well within the 

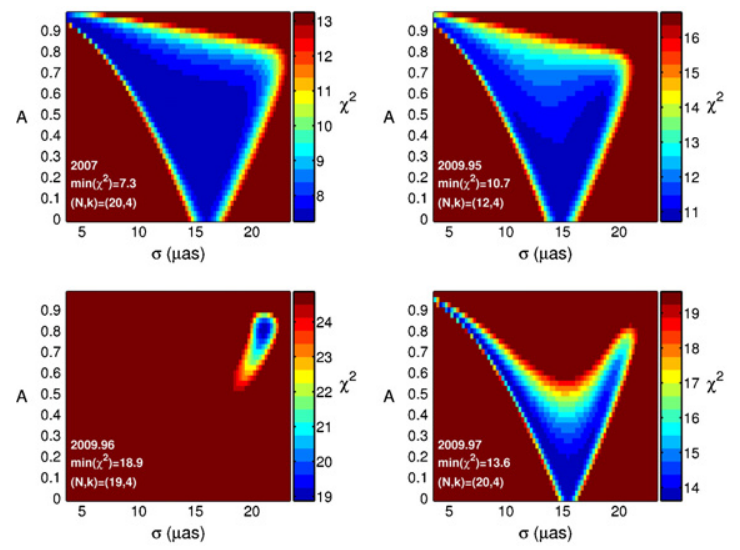

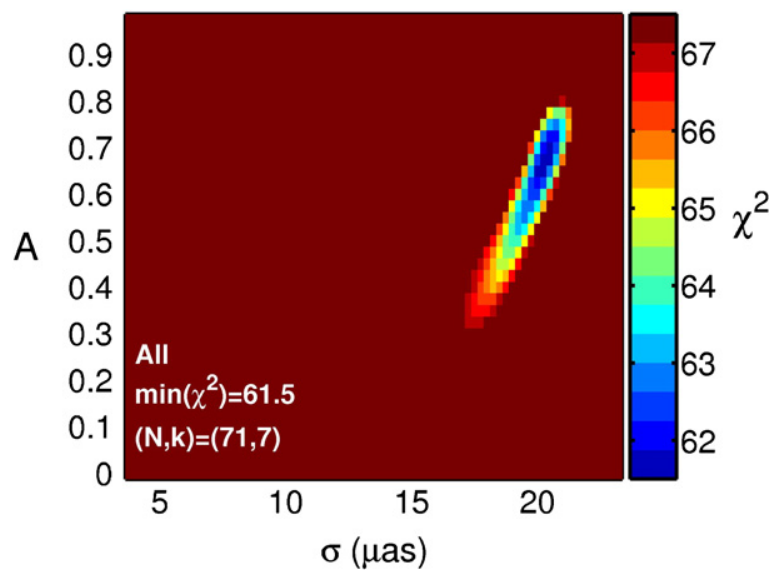

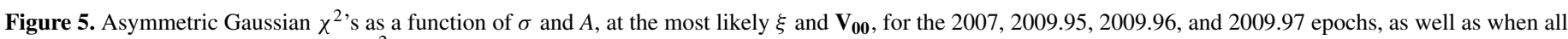

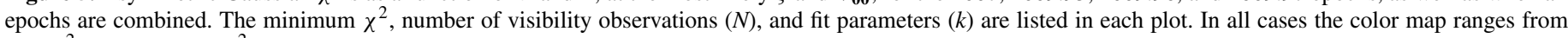
$\min \left(\chi^{2}\right)$ (blue) to $\min \left(\chi^{2}\right)+6$ (red)

(A color version of this figure is available in the online journal.)

Table 1

Model Fitting Results Summary

\begin{tabular}{|c|c|c|c|c|c|c|c|c|c|c|c|c|}
\hline Model & $k$ & $\chi^{2}$ & DoF & $\chi^{2} / \mathrm{DoF}$ & $V_{00}^{2007}$ & $V_{00}^{2009.95}$ & $V_{00}^{2009.96}$ & $V_{00}^{2009.97}$ & $\mathrm{BIC}$ & $w_{i, \mathrm{BL} 06}^{\mathrm{BIC}}$ & $\mathrm{AIC}$ & $w_{i, \mathrm{BL} 06}^{\mathrm{AIC}}$ \\
\hline \multicolumn{13}{|c|}{ Estimated Errors } \\
\hline Symmetric Gaussian & 5 & 76.78 & 66 & 1.16 & 2.37 & 2.08 & 2.03 & 2.88 & 98.1 & $5 \times 10^{-4}$ & 87.7 & $8 \times 10^{-5}$ \\
\hline Asymmetric Gaussian & 7 & 61.50 & 64 & 0.961 & 2.53 & 2.25 & 2.23 & 3.06 & 91.3 & $1 \times 10^{-2}$ & 77.3 & $1 \times 10^{-2}$ \\
\hline BL06 & 7 & 53.09 & 64 & 0.830 & 2.45 & 2.18 & 2.16 & 3.00 & 82.9 & 1 & 68.9 & 1 \\
\hline \multicolumn{13}{|c|}{ Implied Errors } \\
\hline Symmetric Gaussian & 5 & 92.51 & 66 & 1.40 & 2.37 & 2.08 & 2.03 & 2.88 & 114 & $4 \times 10^{-5}$ & 103 & $9 \times 10^{-6}$ \\
\hline Asymmetric Gaussian & 7 & 74.10 & 64 & 1.16 & 2.53 & 2.25 & 2.23 & 3.06 & 104 & $6 \times 10^{-3}$ & 89.9 & $6 \times 10^{-3}$ \\
\hline BL06 & 7 & 63.97 & 64 & 1.00 & 2.45 & 2.18 & 2.16 & 3.00 & 93.8 & 1 & 79.7 & 1 \\
\hline
\end{tabular}

single-epoch uncertainty (defined by the region in which $\chi^{2}$ is within unity of the minimum value). In particular, there are no trends distinguishing either the much earlier 2007 epoch or the considerably brighter 2009.97 epoch. Thus, we conclude that the characteristic size of Sgr A* did not vary substantially from one epoch to the next, despite considerably changes in its luminosity.

The best-fit intrinsic source size is $\sigma=15.8 \pm 0.2 \mu$ as (FWHM $=37.2 \pm 0.5 \mu$ as) with the flux normalization provided in Table 1, ranging from $2.07 \mathrm{Jy}$ to $2.81 \mathrm{Jy}$ over the various epochs. The associated reduced- $\chi^{2}$ is 1.16 , with 66 degrees of freedom, which while somewhat large is still consistent with fitting the visibilities given the $\gtrsim 20 \%$ uncertainties in the error estimates themselves. Upon convolving with the interstellar electron scattering, which along the general direction of the baselines SMT-JCMT and CARMA-JCMT baselines has an FWHM of width of $22 \mu \mathrm{as}$, we find the FWHM of the broadened image is $43.2 \pm 0.6 \mu$ as. This is in excellent agreement with the inferred size of $43_{-3}^{+5}$ found by Doeleman et al. (2008) on the basis of the 2007 epoch alone. ${ }^{7}$ It is also in excellent agreement with the inferred sizes of $41.3_{-1.4}^{+1.8}, 44.4_{-1}^{+1}$, and $42.6_{-1}^{+1}$ found by Fish et al. (2011) for epochs 2009.95, 2009.96, and 2009.97, respectively. ${ }^{7}$ The associated intrinsic and scatter-broadened images with the associated visibilities are shown in the left, center, and right panels of the top row of Figure 3.

\footnotetext{
Here we have quoted the $1 \sigma$ errors upon their result in order to provide a direct comparison.
}

\subsection{Asymmetric Gaussian and Image Anisotropy}

During the 2007 epoch the mm-VLBI visibilities are concentrated nearly exclusively along a single line, oriented nearly east-west (see the top left panel of Figure 1). However, upon including the 2009 epochs, the portion of the $u-v$ plane sampled covers a bow-tie-shaped region with opening angle $26^{\circ}$ (see the bottom-center panel of Figure 1). While this is insufficient to generate an image directly, the coverage is sufficient to address the gross angular structure of Sgr A*'s image. Where the symmetric Gaussian provides a phenomenological way in which to estimate the typical size of Sgr A*'s emitting region, an asymmetric Gaussian can begin to probe its symmetry.

Figure 5 shows the minimum $\chi^{2}$ (or equivalently, the maximum likelihood) as a function of the average size, $\sigma$, and anisotropy parameter, $A$. The four left panels show this for the individual epochs, while the large right panel shows this for the combined data set. Unlike the symmetric Gaussian model, the likely regions have somewhat different morphologies. This is due almost entirely to the different coverage of the $u-v$ plane during the various epochs (for example, the likely regions for epochs 2007 and 2009.95 are similar because the $u-v$ coverage during these epochs is similar). Nevertheless, the region preferred by the combined data sets is present in all cases, implying that as with the symmetric Gaussian all epochs are consistent with a single underlying image morphology. During this time the flux normalization of the compact component varied from $2.23 \mathrm{Jy}$ (2009.96) to 3.06 Jy (2009.97).

For all epochs the reduced- $\chi^{2}$ is nearly unity, ranging from 0.45 (2007) to 1.34 (2009.95), implying that adequate 

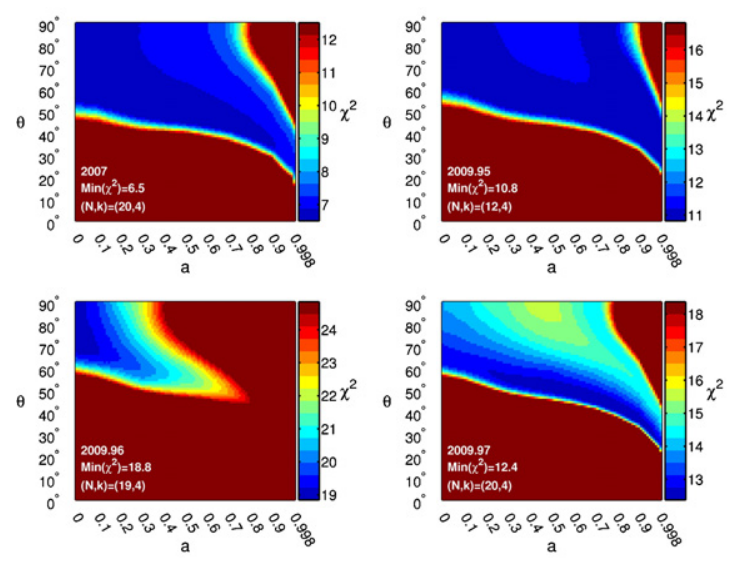

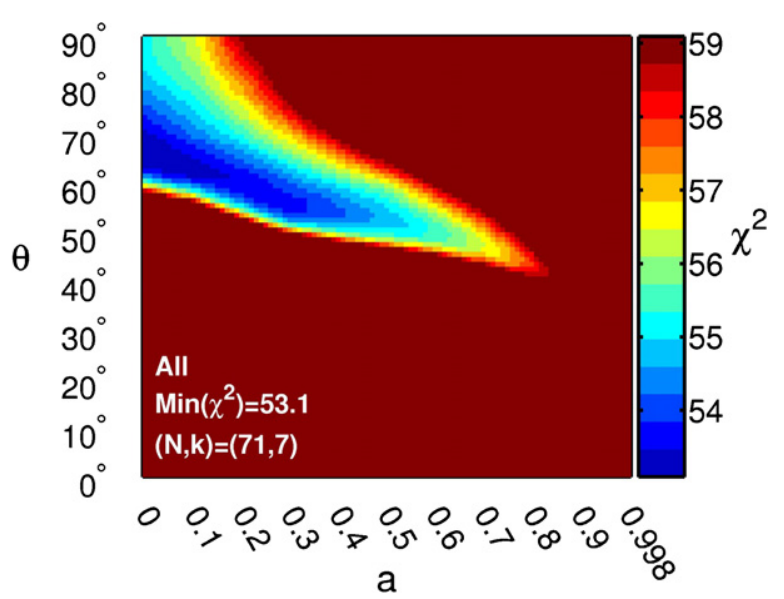

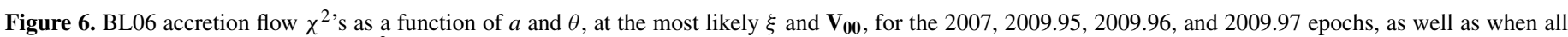

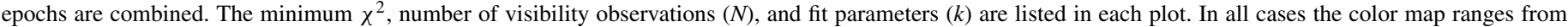
$\min \left(\chi^{2}\right)$ (blue) to $\min \left(\chi^{2}\right)+6$ (red).

(A color version of this figure is available in the online journal.)

fits exist. The most likely configuration is highly asymmetric, with $\sigma=20.5_{-0.8-1.3}^{+0.3+0.5} \mu \mathrm{as}, A=0.70_{-0.1-0.18}^{+0.03+0.05}$, and $\xi=$ $-19^{\circ}+3^{\circ}+6^{\circ}-2^{\circ}$, corresponding to a major-minor axis ratio of more than $2.4_{-0.4-0.6}^{+0.2+0.3}$, with symmetric models highly disfavored. The resulting FWHMs of the minor and major axes are then $37 \pm 1 \mu$ as and $88 \pm 9 \mu$ as, though these are significantly correlated due to the substantially larger fractional error on $A$ in comparison to that on $\sigma$. The intrinsic image, scatterbroadened image, and associated visibilities of the most likely configuration is shown in the middle row of Figure 3.

The reduced $-\chi^{2}$ for the combined data set is 0.961 , considerably lower than that found for the symmetric case, and again implying that a single, static image is consistent with fitting the visibilities. As described in Section 4, a decrease in $\chi^{2}$ is expected given the addition of two parameters. However, the various ICs, given in Table 1, provide a means for identifying lower $\chi^{2}$ that are significant. The best-fit asymmetric model has a BIC that is 6.8 lower than the best-fit symmetric model, and an AIC that is 10.4 lower than the best-fit symmetric model. These provide "strong" evidence against symmetric models for the image of Sgr A*, ruling these out at $2.6 \sigma$ (BIC) and $3.2 \sigma$ (AIC) levels, in terms of the relative significance. That is, despite the limited visibility coverage in the $u-v$ plane, the existing mm-VLBI observations can conclusively detect asymmetric structure in $\operatorname{Sgr} \mathrm{A}^{*}$.

\subsection{Accretion Flow and Implications of Physics}

The images of the BL06 model, described in Section 3.1, are characterized by asymmetric crescents. These arise due to the combination of gravitational lensing, the relativistic orbital motion, and the opacity of the underlying accretion flow. The size and extent of the crescent depends upon the spin and inclination of the system, with nearly face-on disks ( small $\theta$ ) appearing annular.

Because we only have access to the visibility magnitudes, configurations rotated by $180^{\circ}$ are indistinguishable, imposing an unavoidable ambiguity upon any results. In addition, despite opacity, the images exhibit nearly exact symmetry between configurations viewed from above the equatorial plane (i.e., $\theta<90^{\circ}$ ) and those viewed from below the equatorial plane (i.e., $\theta>90^{\circ}$ ) at equal inclinations. As a consequence, there is also an ambiguity in the line-of-sight component of the spin vector. For this reason, here we discuss only $0^{\circ} \leqslant \theta \leqslant 90^{\circ}$. However we have performed the analysis for $90^{\circ} \leqslant \theta \leqslant 180^{\circ}$ as well, finding no statistically significant differences in the parameter estimates.

Figure 6 shows the minimum $\chi^{2}$ (maximum likelihood) as a function of spin, $a$, and viewing angle, $\theta$. As with Figure 5, the four panels on the left show this for the individual epochs, while the large panel on the right shows $\chi^{2}$ for the combined data set. The morphology is similar in all cases, with the different $u-v$ coverage during the different epochs manifesting itself primarily in the size of the likely region. As a result, we conclude that again the four epochs are consistent with a single underlying image morphology. Over all of the observations the flux normalization varied from $2.16 \mathrm{Jy}(2009.96)$ to $3.00 \mathrm{Jy}$ (2009.97) and is at all times sufficiently close to the value of $2.5 \mathrm{Jy}$ used to compute the images of the accretion flow. The intrinsic image, scatter-broadened image, and corresponding visibilities of the most probable configuration (not necessarily the lowest $\chi^{2}$, see Section 6) is shown in the bottom row of Figure 3.

The reduced $-\chi^{2}$ of 0.830 found for the BL06 models is the smallest of any model we consider, and again implies that the images provide adequate descriptions of the data. Again we may assess the significance of the improvement over the phenomenological Gaussian models by appealing to the ICs described in Section 4. In this case the BIC and AIC are given by 82.9 and 68.9 , respectively. These are much lower than those from the symmetric Gaussian, providing "decisive" evidence against symmetric configurations. Both are also below those of the asymmetric Gaussian by 8.4 (since the number of parameters is the same in both models), implying "strong" evidence for the physically motivated accretion flow model in contrast to the phenomenological asymmetric Gaussian. This corresponds to a $2.9 \sigma$ confidence level in terms of the relative significance of the two models; i.e., the physically motivated accretion models are more than 67 times as likely as the phenomenological asymmetric Gaussian models, and more than $2 \times 10^{3}$ times as likely as symmetric Gaussian models. However, for two reasons this actually understates the case.

First, the reduced $-\chi^{2}$ of the BL06 models is significantly less than unity. Indeed, with 64 degrees of freedom we expect a $\chi^{2}$ lower than that obtained (53.09) only $17 \%$ of the time. There are three reasons this may have occurred: we may simply be lucky, 

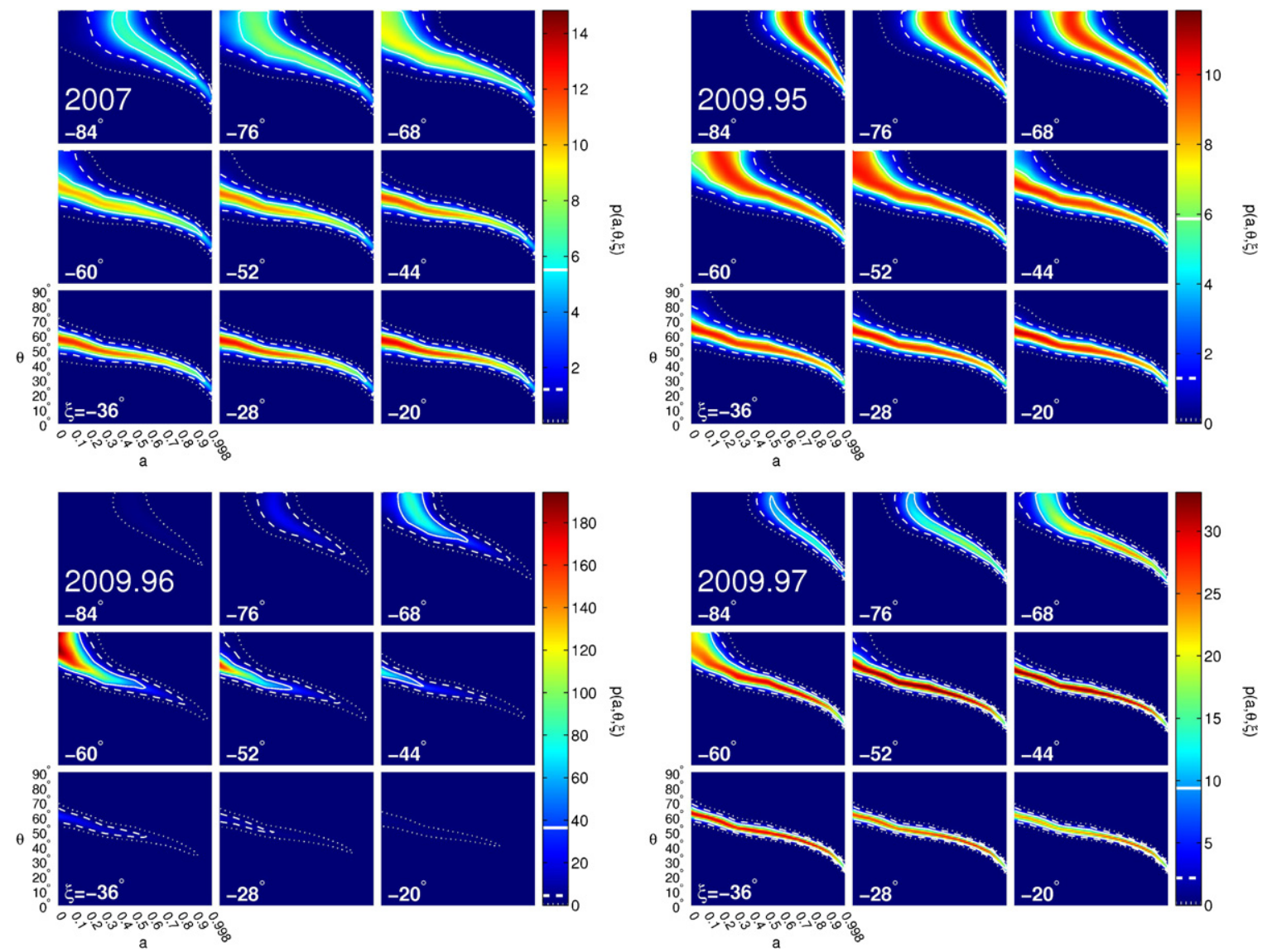

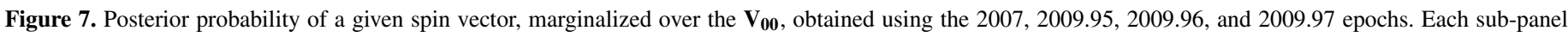

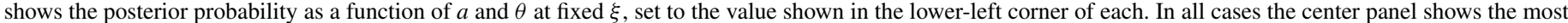

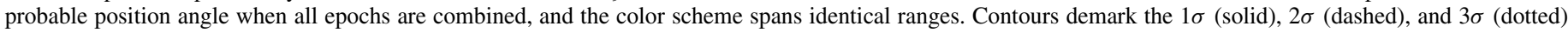
regions, defined by cumulative probability.

(A color version of this figure is available in the online journal.)

in which case there is nothing significant about the small $\chi^{2}$ value; we may be overfitting the data; the error estimates on the individual visibility magnitudes by be too large. We may safely reject second possibility given both the large number of degrees of freedom and the fact that the BL06 models have the same number of parameters as the asymmetric Gaussian. While, the probability of obtaining the measured $\chi^{2}$ is not sufficiently low to exclude chance, we may nonetheless obtain an estimate for the "true" errors by considering the third possibility: asserting that the error estimates are overestimated by a moderate amount. On the other hand, we may measure the "true" errors by assuming that the BL06 model gives a sufficiently close approximation to the true image flux by renormalizing them until the reduced- $\chi^{2}$ is unity. This requires a roughly $10 \%$ reduction in the errors quoted in Section 2. This in turn alters the $\chi^{2}$, BIC, and AIC for the other models as well. These values are listed under the "Implied Errors" section of Table 1. The net effect is to increase the significance with which the asymmetric Gaussian is ruled out relative to the BL06 accretion model to $3.2 \sigma$. While this most likely simply provides a measure of the uncertainty of the relative significances, it suggests that the "true" significance with which the phenomenological models can be ruled out is larger than implied by the original error estimates alone.

Second, the accretion flow models should generally be preferred on the basis of their motivation alone. That is, there is a strong prior in favor of physically motivated models by virtue of their design and connection to an existing body of knowledge. Furthermore, in the case of Sgr A* this includes the fact that these models were constrained to fit the pre-existing spectral data as well, something that is only possible because the physics governing the accretion flow provides a means to relate the properties of images at different wavelengths.

The fact that the BL06 models are significantly preferred by the mm-VLBI data implies that we can now distinguish phenomenological and physically motivated models on the basis of mm-VLBI observations alone. Since the most prominent features of the accretion flow image are due to the generic properties of black hole accretion flows, namely, the spacetime and orbital motion, this is likely to be robust among all RIAF models for Sgr $A^{*}$. That is, even with the extremely sparse $u-v$ coverage presently available it is already possible to probe signatures of general relativity and accretion physics in the image itself.

\section{ESTIMATING BLACK HOLE SPIN}

Following Broderick et al. (2009) we produce posterior probability distributions for the parameters defining the BL06 model, assuming a flat prior on $a$ and isotropic priors upon the spin direction, and marginalizing over the $\mathbf{V}_{\mathbf{0 0}}$. For the three-dimensional parameter space defining the vector black hole spin, $(a, \theta, \xi)$, this is done for each epoch individually (Figure 7) as well as for the combined data set (Figure 8). For each epoch we show the probability distribution for the same 


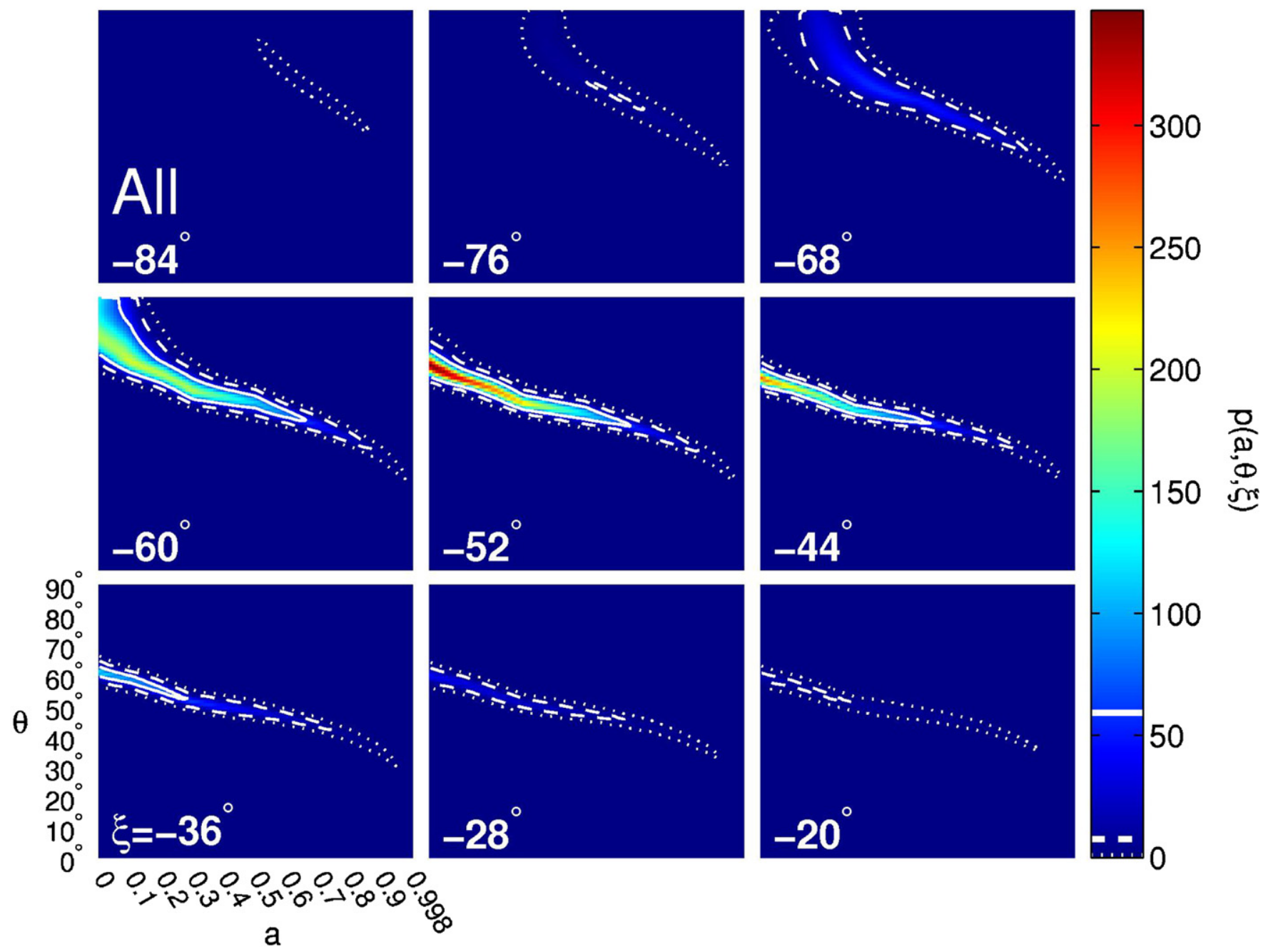

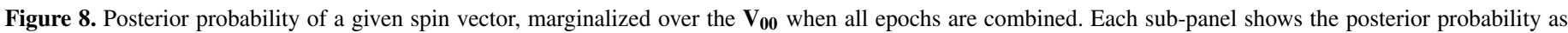

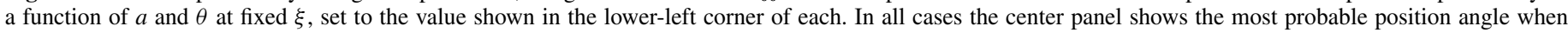

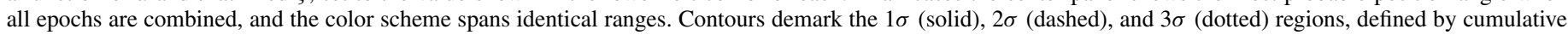
probability.

(A color version of this figure is available in the online journal.)

position angle slices, defined such that the most probable values from the combined data set are exhibited in the central panel. In all cases we define $1 \sigma, 2 \sigma$, and $3 \sigma$ contours (shown by the solid, dashed, and dotted lines, respectively) in terms of the cumulative probability: the contours lie upon the probability densities above which $68 \%, 95 \%$, and $99.7 \%$ of the total probability lie, respectively (for an explicit definition, see Equation (20) of Broderick et al. 2009). The probability densities are normalized to their average value, i.e., if all points were equally probable the probability density would be unity.

Note that the way in which we have chosen the slices in $\xi$ in Figure 7 does not capture the most likely configuration based upon the 2007 epoch alone (shown in Broderick et al. 2009). Nevertheless, the regions shown are well within the $1 \sigma$ region from that epoch. Here we explicitly see that all epochs produce consistent estimates for the spin, with varying degrees of statistical strength.

The combined data set dramatically restricts the parameter estimates to a narrow sliver in the three-dimensional spin parameter space. The most probable values for the spin are

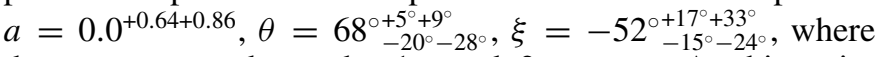
the errors quoted are the $1 \sigma$ and $2 \sigma$ errors. At this point the probability density is roughly 350 times that of the average value. In practice, these quantities are much more tightly correlated with

$$
\theta \simeq 68^{\circ}-42^{\circ} a \pm 3^{\circ} \pm 5^{\circ}
$$

Note that these are degenerate with solutions for which $\xi$ differs by $180^{\circ}$, i.e., $\xi=128^{\circ}{ }_{-15^{\circ}-24^{\circ}}$, and for which the line-of-sight component of the spin is reversed, i.e., $\theta=112^{\circ}+20^{\circ}+28^{\circ}$.

We do not attempt to determine the systematic uncertainties associated with selecting a particular accretion model. However, we note that a number of efforts to fit alternative accretion flow models to the 2007 epoch have reached consistent results despite differences in the models, suggesting that these results are robust. Furthermore, the quality of the fits to the mmVLBI visibility and spectral data, concurrently, suggests that the features of the BL06 model responsible for determining the spectral and image properties are generic, and are therefore insensitive to the particulars of the accretion flow models. However, full studies of the systematic errors associated with the particular choices made for the accretion flow properties and the underlying spacetimes are now justified. While beyond the 

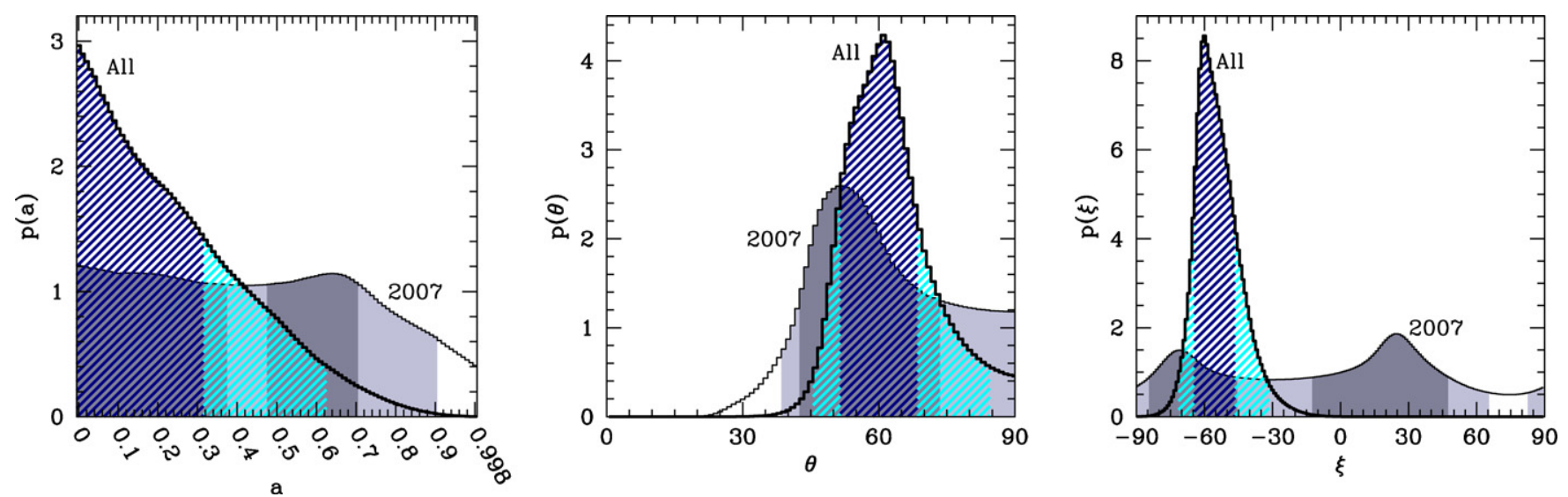

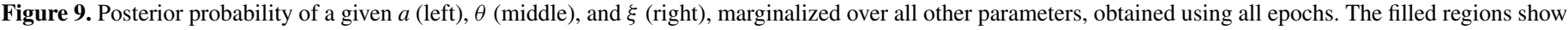
the $1 \sigma$ (dark) and $2 \sigma$ (light) regions, defined by cumulative probability.

(A color version of this figure is available in the online journal.)

purview of the present paper, we will report upon such efforts elsewhere.

Probability distributions for each of the spin parameters, marginalized over all others, are shown in Figure 9. In addition to the present case, these are also shown for the analysis of the 2007 epoch for comparison. In these the $1 \sigma$ and $2 \sigma$ ranges, defined by the cumulative probability, are also shown. In all cases the marginalized probability distributions from the combined data set are much more narrowly peaked than their 2007 epoch counterparts. Nevertheless, they are all consistent at the $1 \sigma$ level with those obtained from the 2007 epoch alone.

It is now possible to exclude $a>0.62$ at the $2 \sigma$ level, with $a=0^{+0.32+0.62}$, substantially preferring non-spinning models. Thus, the high-spin island seen in Figure 7 of Broderick et al. (2009) is now eliminated. Similarly, the position angle is now very clearly constrained, choosing the solution less favored by the 2007 epoch data (though still consistent at the $1 \sigma$ level). In

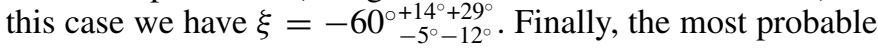
viewing angle is $\theta=61^{\circ}{ }_{-9^{\circ}-14^{\circ}}^{\circ}$. This is somewhat higher than the most likely value from the 2007 epoch alone, though well within the $1 \sigma$ uncertainty.

These estimates for the orientation of the spin vector are in good agreement with a number of other efforts to estimate the properties of Sgr A*'s accretion flow. Estimates based upon fitting longer wavelength observations with numerical models of RIAFs produce position angles and inclination estimates with large uncertainties, though these are nevertheless consistent with the results obtained here (Huang et al. 2007). It is also in excellent agreement with more recent attempts to probe the spin orientation using the mm-VLBI data from the 2007 epoch (Huang et al. 2009; Dexter et al. 2010). As before it is not possible to assess consistency with models that employ qualitatively different plasma distributions near the black hole (e.g., Markoff et al. 2007), though they tend to imply similarly large viewing angles.

We find similar spin orientations to those inferred from modeling of infrared polarization observations of Sgr $\mathrm{A}^{*}$ 's flaring emission, though in this case the uncertainties are considerable (e.g., Meyer et al. 2007). Similarly, we find consistency with the spin directions obtained from modeling the spectrum and polarization using general relativistic MHD simulations, despite preferring significantly smaller spin magnitudes (Shcherbakov et al. 2010).
Unlike the estimates in Broderick et al. (2009), there is no longer any allowed solution for the spin vector that aligns with either of the reported stellar disks in the inner $0.2 \mathrm{pc}$ of the Galactic center (Genzel et al. 2003). However, our revised position angle estimates are consistent with being aligned with the X-ray feature reported in Muno et al. (2008), bolstering the interpretation of this as related to a possible jet. Note, however, this interpretation may be inconsistent with the low spin magnitudes we prefer.

\section{OPTIMIZING FUTURE OBSERVATIONS}

The constraints upon the accretion-model parameters obtained in the previous sections have implications for future mm-VLBI experiments. With these it is possible both to make predictions for the expected visibilities on the various possible baselines, as well as identify which baselines are most likely to provide substantial improvements to the BL06 model parameter estimation. To estimate these, here we compute the average visibility amplitudes as well as the variance associated with the uncertainty in the model parameters, weighted by the posterior probability distributions we have obtained using the combined mm-VLBI data set, following the method of Fish et al. (2009).

The probability-weighted mean visibility profile of the scatter-broadened $230 \mathrm{GHz}$ emission from Sgr A* is elongated in the $(+u,+v)$ direction (Figure 10). For the moderately high values of $\theta$ favored by the mm-VLBI data, the intensity profile is dominated by Doppler-boosted emission on the approaching side of the accretion flow (e.g., in the northeast of lower-left panel of Figure 3). This portion of the emission is elongated parallel to the projected direction of the black hole spin vector. Since the effective size of the emission is larger along the projected spin axis, the correlated flux density falls off faster with baseline length for baselines that are sensitive to structure in this direction than in the perpendicular direction. Our estimates of RIAF parameters suggest that a mm-VLBI baseline oriented southwest-northeast will detect more correlated flux density than an equal-length baseline oriented southeast-northwest.

The standard deviation of the visibility amplitudes provides an estimate of which baselines would provide maximal additional constraints on RIAF model parameters (assuming equal sensitivity at all sites). Previous computations based on the 2007 epoch of data indicated that the largest scatter occurred at baseline lengths of approximately $3 \mathrm{G} \lambda$ and with orientations 

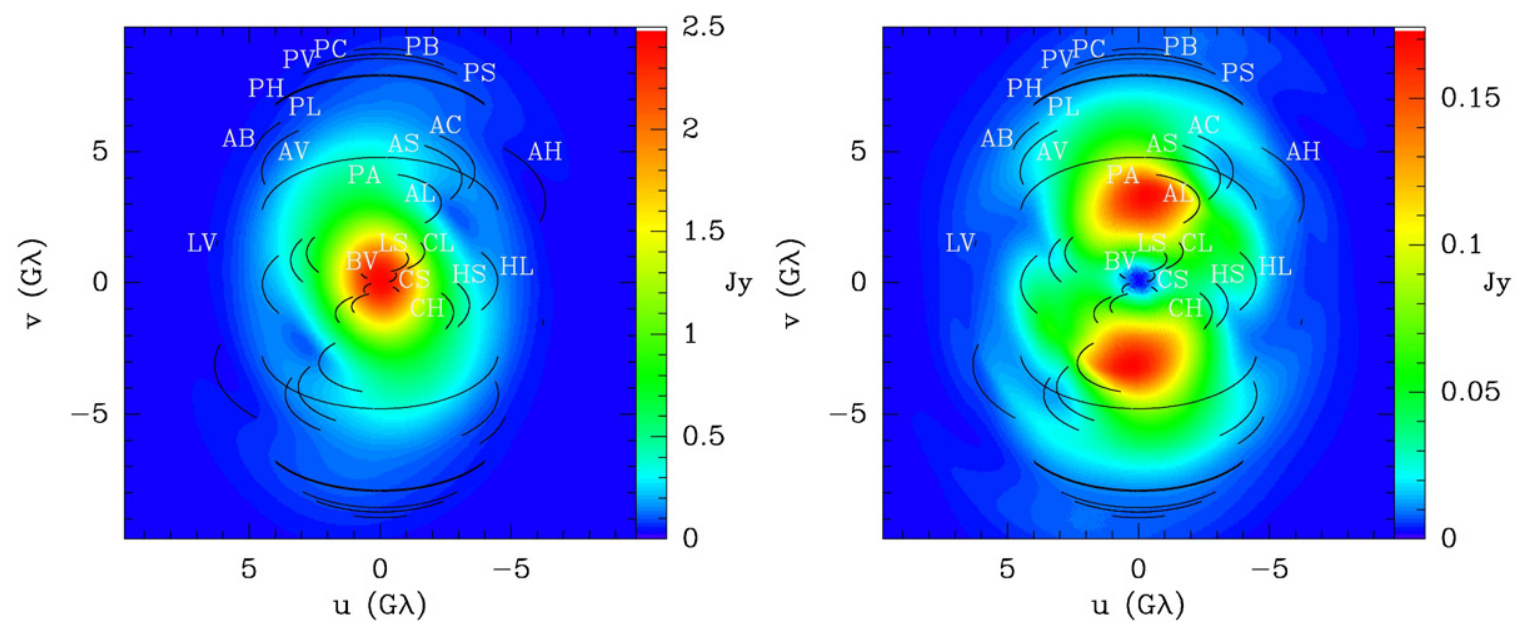

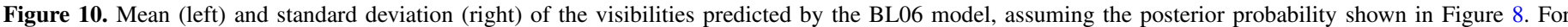

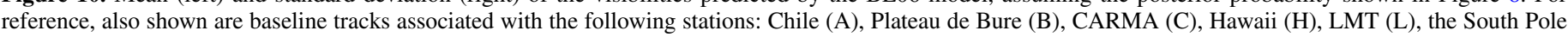
(P), SMT (S), and Pico Veleta (V).

(A color version of this figure is available in the online journal.)

perpendicular to the Hawaii-SMT baseline (Fish et al. 2009). These findings still hold in light of the 2009 data.

Among possible observing baselines in the next few years, Chile-SPT and Chile-LMT probe the region of highest standard deviation, followed by baselines between the LMT and the continental US. Our model suggests that the LMT-SMT and LMT-CARMA baselines will detect well over $1 \mathrm{Jy}$. The increased sensitivity provided by phasing many ALMA dishes together will be decisive for RIAF modeling efforts using the baselines to Chile, as the probability-weighted mean visibility amplitudes are $\gtrsim 0.1 \mathrm{Jy}$ on the Chile-SMT baseline, $\lesssim 0.1 \mathrm{Jy}$ over most of the $(u, v)$ track of the Chile-CARMA baseline, and smaller still on the longer baselines to Chile. Assuming nominal antenna characteristics at ALMA and SMT, a reasonable extension to VLBI bandwidths of $4 \mathrm{GHz}$ over the next few years, and summing 25 ALMA dishes, the SMT-ALMA baseline will detect $0.1 \mathrm{Jy}$ of correlated flux from $\mathrm{Sgr} \mathrm{A}^{*}$ with a signal to noise of $\sim 25$ in only 3 minutes. By comparison, the standard deviation of the predicted model visibility amplitudes is several $\times 10 \mathrm{mJy}$ on these baselines, corresponding to tens of percent in model amplitude predictions. Further mm-VLBI data, either in the form of higher sensitivity on existing baselines or detections on new baselines, will both reduce these uncertainties and test the RIAF model with increasing rigor.

\section{CONCLUSIONS}

The significantly increased number of long-baseline visibilities, significantly larger signal-to-noise, and improved north-south coverage of the $2009 \mathrm{~mm}$-VLBI observations have already paid substantial dividends in the estimation of the properties of Sgr $\mathrm{A}^{*}$. This is despite the fact that only three independent mm-VLBI stations (JCMT, CARMA, SMT) were used, and therefore the $u-v$ plane remains extremely sparsely populated, with long baselines primarily in the east-west direction. Constraints upon the black hole spin have improved dramatically in all cases, with the spin and viewing angle becoming tightly correlated, with the most probable configuration, $a=0.0^{+0.64+0.86}$, $\theta=68_{-20^{\circ}-28^{\circ}}, \xi=-52^{\circ}{ }_{-15^{\circ}-24^{\circ}+33^{\circ}}^{\circ}$ being roughly 350 times as likely as the average probability density, and 25 times as likely as the most probable configuration reported in Broderick et al. (2009).
Despite the limited north-south coverage, the $2009 \mathrm{~mm}$ VLBI data conclusively exclude symmetric Gaussian models for the source. The preferred asymmetric Gaussian has a major-minor axis ratio of $2.4_{-0.4-0.6}^{+0.2+0.3}$, oriented with the major axis oriented $-19^{\circ}+3^{\circ}+6^{\circ}$ 。 east of north. This implies major and minor axis FWHMs of $37 \pm 1 \mu$ as and $88 \pm 9 \mu$ as, with the symmetric case excluded at $3.9 \sigma$ significance. Note that this orientation is not aligned with any particular feature in Sgr A*'s vicinity or the properties of the intervening interstellar electron scattering screen.

It is natural to give physically motivated models a prior bias over phenomenological models. Nevertheless, even when physically motivated accretion models are compared with asymmetric Gaussian models are weighted equally, the accretion models provide a significantly better fits to the mm-VLBI data. Based upon both the BIC and AIC we find strong evidence in favor of the accretion model, corresponding to a posterior probability 67 to 160 times larger than that of the most likely asymmetric Gaussian model. This is particularly striking given the simplicity of the accretion model we consider, suggesting that the image depends primarily upon the gross dynamical and geometric properties of the system: the orbital motion of the accreting material and the strong gravitational lensing by the black hole. In any case, it is clear that we have now entered the era of studying accretion and black hole physics with mm-VLBI.

Our best-fit accretion model requires a black hole spin of $a=0.0^{+0.64+0.86}$ viewed at an angle of $\theta=68_{-20^{\circ}-28^{\circ}}^{\circ}$, oriented at a position angle of $\xi=-52^{\circ}+17^{\circ}+33^{\circ}-24^{\circ}$. Ambiguities due to the fact that only amplitudes of the visibilities were measured and the near symmetry of the accretion-model images produce degeneracies corresponding to $\theta \leftrightarrow 180^{\circ}-\theta$ and $\xi \leftrightarrow \xi+180^{\circ}$, independently. The detection of closure phases will eliminate the ambiguity in position angle.

During all of the mm-VLBI epochs presently available, three or fewer VLBI stations were employed, providing at most three baselines. In practice the two long baselines are nearly collinear, aligned predominantly east-west. The resulting sparse coverage within $u-v$ plane is the primary factor limiting the estimation of black hole and accretion flow parameters. Therefore, despite the success attained thus far, there is a considerable opportunity to improve the constraints upon $\mathrm{Sgr} \mathrm{A}^{*}$ and its accretion 
flow dramatically in the near future by including additional VLBI stations. The Event Horizon Telescope is a millimeter and submillimeter wavelength VLBI network whose goal is to observe, image, and time resolve structures near the black hole event horizon (Doeleman et al. 2009a). Over the next few years, new sites will join the current array, and sensitivities of critical baselines (e.g., those found in Section 7) will be enhanced through technical developments to widen bandwidths and phase together multiple dishes at millimeter array sites (e.g., CARMA, SMA, ALMA). An important result is that within the next few years, interferometric phase information on long VLBI baselines will become available through robust measurement of the closure phase (Doeleman et al. 2009b), which will provide important new constraints on models of Sgr A*.

This work was supported in part by NSF grants AST0907890, AST-0807843, and AST-0905844, and NASA grants NNX08AL43G and NNA09DB30A. A.E.B. gratefully acknowledges the support of the Beatrice D. Tremaine Fellowship.

\section{REFERENCES}

Agol, E. 2000, ApJ, 538, L121

Aitken, D. K., Greaves, J., Chrysostomou, A., Jenness, T., Holland, W., Hough, J. H., Pierce-Price, D., \& Richer, J. 2000, ApJ, 534, L173

Beckwith, K., Hawley, J. F., \& Krolik, J. H. 2008, ApJ, 678, 1180

Blandford, R. D., \& Begelman, M. C. 1999, MNRAS, 303, L1

Bower, G. C., Goss, W. M., Falcke, H., Backer, D. C., \& Lithwick, Y. 2006, ApJ, 648, L127

Bower, G. C., Wright, M. C. H., Falcke, H., \& Backer, D. C. 2001, ApJ, 555, L103

Bower, G. C., Wright, M. C. H., Falcke, H., \& Backer, D. C. 2003, ApJ, 588, 331

Broderick, A., \& Blandford, R. 2004, MNRAS, 349, 994

Broderick, A. E. 2006, MNRAS, 366, L10

Broderick, A. E., Fish, V. L., Doeleman, S. S., \& Loeb, A. 2009, ApJ, 697, 45

Broderick, A. E., \& Loeb, A. 2006a, ApJ, 636, L109

Broderick, A. E., \& Loeb, A. 2006b, MNRAS, 367, 905

Burnham, K. P., \& Anderson, D. R. 2002, Model Selection and Multimodal Inference (New York: Springer)

Burnham, K. P., \& Anderson, D. R. 2004, Sociol. Methods Res., 33, 261

Cuadra, J., Nayakshin, S., \& Martins, F. 2008, MNRAS, 383, 458

De Villiers, J., Hawley, J. F., Krolik, J. H., \& Hirose, S. 2005, ApJ, 620, 878

Dexter, J., Agol, E., Fragile, P. C., \& McKinney, J. C. 2010, ApJ, 717, 1092
Doeleman, S., et al. 2009a, Astro2010: The Astronomy and Astrophysics Decadal Survey, Science White Papers, No., 68

Doeleman, S. S., Fish, V. L., Broderick, A. E., Loeb, A., \& Rogers, A. E. E. 2009b, ApJ, 695, 59

Doeleman, S. S., et al. 2008, Nature, 455, 78

Falcke, H., \& Markoff, S. 2000, A\&A, 362, 113

Fish, V. L., Broderick, A. E., Doeleman, S. S., \& Loeb, A. 2009, ApJ, 692, L14

Fish, V. L., et al. 2011, ApJ, 727, L36

Genzel, R., et al. 2003, ApJ, 594, 812

Ghez, A. M., et al. 2008, ApJ, 689, 1044

Gillessen, S., Eisenhauer, F., Fritz, T. K., Bartko, H., Dodds-Eden, K., Pfuhl, O., Ott, T., \& Genzel, R. 2009a, ApJ, 707, L114

Gillessen, S., Eisenhauer, F., Trippe, S., Alexander, T., Genzel, R., Martins, F., \& Ott, T. 2009b, ApJ, 692, 1075

Hawley, J. F., \& Krolik, J. H. 2006, ApJ, 641, 103

Huang, L., Cai, M., Shen, Z., \& Yuan, F. 2007, MNRAS, 379, 833

Huang, L., Takahashi, R., \& Shen, Z. 2009, ApJ, 706, 960

Jones, T. W., \& O'Dell, S. L. 1977, ApJ, 214, 522

Krichbaum, T. P., et al. 1998, A\&A, 335, L106

Kurosawa, R., \& Proga, D. 2009, ApJ, 693, 1929

Liddle, A. R. 2007, MNRAS, 377, L74

Loeb, A., \& Waxman, E. 2007, J. Cosmol. Astropart. Phys., JCAP03(2007)011

Macquart, J., Bower, G. C., Wright, M. C. H., Backer, D. C., \& Falcke, H. 2006, ApJ, 646, L111

Markoff, S., Bower, G. C., \& Falcke, H. 2007, MNRAS, 379, 1519

Marrone, D. P. 2006, PhD thesis, Harvard Univ.

Marrone, D. P., Moran, J. M., Zhao, J., \& Rao, R. 2006, J. Phys.: Conf. Ser., 54, 354

Marrone, D. P., Moran, J. M., Zhao, J., \& Rao, R. 2007, ApJ, 654, L57

McKinney, J. C. 2006, MNRAS, 368, 1561

McKinney, J. C., \& Blandford, R. D. 2009, MNRAS, 394, L126

Meyer, L., Schödel, R., Eckart, A., Duschl, W. J., Karas, V., \& Dovčiak, M. 2007, A\&A, 473, 707

Mościbrodzka, M., Gammie, C. F., Dolence, J. C., Shiokawa, H., \& Leung, P. K. 2009, ApJ, 706, 497

Muno, M. P., Baganoff, F. K., Brandt, W. N., Morris, M. R., \& Starck, J. 2008, ApJ, 673, 251

Narayan, R., Mahadevan, R., Grindlay, J. E., Popham, R. G., \& Gammie, C. 1998, ApJ, 492, 554

Penna, R. F., McKinney, J. C., Narayan, R., Tchekhovskoy, A., Shafee, R., \& McClintock, J. E. 2010, MNRAS, 408, 752

Petrosian, V., \& McTiernan, J. M. 1983, Phys. Fluids, 26, 3023

Quataert, E., \& Gruzinov, A. 2000, ApJ, 545, 842

Sharma, P., Hammett, G. W., Quataert, E., \& Stone, J. M. 2006, ApJ, 637, 952

Sharma, P., Quataert, E., Hammett, G. W., \& Stone, J. M. 2007, ApJ, 667, 714

Shcherbakov, R. V., Penna, R. F., \& McKinney, J. C. 2010, arXiv:1007.4832

Takeuchi, T. T. 2000, Ap\&SS, 271, 213

Tchekhovskoy, A., Narayan, R., \& McKinney, J. C. 2010, ApJ, 711, 50

Yuan, F., Markoff, S., \& Falcke, H. 2002, A\&A, 383, 854

Yuan, F., Quataert, E., \& Narayan, R. 2003, ApJ, 598, 301

Yuan, F., Quataert, E., \& Narayan, R. 2004, ApJ, 606, 894 